\title{
Synthesis of a Continuously Operated Mass-Exchanger Network for a Semiconsecutive Process
}

\author{
Cheng-Liang Chen* and Ying-Jyuan Ciou \\ Department of Chemical Engineering, National Taiwan University, Taipei 10617, Taiwan, Republic of China
}

\begin{abstract}
The startings and endings of available process streams are usually time-dependent for a semiconsecutive (intermittent) process. The objective of this paper is to propose an optimization-based strategy for synthesizing a continuously operated mass-exchanger network (MEN) and its associated storage policy for treating process streams enriched with some undesirable components produced from an intermittently operated process. A periodically partitioned stagewise superstructure is presented for modeling the continuous MEN configuration, as well as the time-dependent operations for collecting process streams into storage tanks and releasing accumulated material into the continuous MEN for subsequent treatment. Not using any heuristics that are based on the concepts of pinch limitation, the proposed superstructure-based representation for the synthesis of continuous MENs for semiconsecutive processes is formulated as a mixed-integer nonlinear program (MINLP) for targeting the total annual cost (TAC). The coke oven gases (COG) problem from the literature is adapted and modified as an illustrative case to demonstrate the applicability of the proposed MEN synthesis method for semiconsecutive processes.
\end{abstract}

\section{Introduction}

A mass-exchange unit conducts the duty of transferring undesirable components from a rich process stream into relatively lean mass-separating agents (MSAs). A massexchange network (MEN) consists of several mass-exchange units arranged in a specific configuration, which simultaneously accomplish the treatment of multiple rich streams, using available process lean streams and/or external MSAs. In comparison to recent remarkable advances on the synthesis of MENs for continuous processes, ${ }^{1-7}$ only limited progress can be found when the process streams are intermittent, i.e., the existence of process streams are time-dependent.

One challenge of treating rich process streams following a semiconsecutive process is that the available inlet streams have different starting and ending times. The subsequent MENs can be operated in two modes. The intermittent MEN mode is to execute the mass-exchange units according to the existence of inlet streams, such as that shown in Figure 1a. Therein, down streams flowing from the intermittent MEN to their succeeding units are still time-dependent streams. The synthesis of intermittent MENs is the subject of one recent article. ${ }^{8}$ However, the operation of such an intermittent MEN is questionable in practice, because of frequent startup and shutdown operations on mass-exchange units. Another alternative is to conduct the mass-exchange duty in a consecutive mode, as shown in Figure $1 \mathrm{~b}$, where part of the surplus streams are accumulated in accompanied storage vessels and/or tanks, and the collected material is released late during deficit periods. In such a case, the MEN can be operated continuously and the succeeding outlets from the exchangers will then become continuous streams.

Until now, only few papers investigate the synthesis problems for intermittent MENs. ${ }^{8-11}$ Therein, Wang and Smith ${ }^{9}$ attempted to apply the pinch technique to maximize the driving force in each of the concentration interval in a time-dependent water network synthesis problem. Foo et al. ${ }^{10}$ used time-dependent composition interval table to study the intermittent MENs

* To whom correspondence should be addressed. Tel.: 886-223636194. Fax: 886-2-23623040. E-mail address: CCL@ntu.edu.tw. synthesis problem involving mass-separating agents (MSAs) other than water. Chen and $\mathrm{Ciou}^{8}$ presented a superstructurebased MINLP formulation for synthesis of intermittent MENs, where the role of storage vessels/tanks are investigated for reducing the consumption of external MSAs. However, the investigation concerning about the synthesis of a consecutive MEN following a semicontinuous process and the associated storage policy is rare in the literature.

The objective of this paper is to extend the formulation of Chen and $\mathrm{Ciou}^{8}$ to the synthesis of continuous MENs following semiconsecutive processes. A stagewise superstructure will be presented and the associated storage policy will be investigated for handling time-dependent streams. One example from the literature, ${ }^{10}$ the coke oven gases (COG) process, will be adopted and be modified into different cases to demonstrate the efficacy of the proposed continuous MEN synthesis method for semiconsecutive processes.

The remainder of this paper is organized as follows. The problem statement for the synthesis of continuous MENs for intermittent processes is given in detail in section 2. A periodically partitioned superstructure is then proposed for sequentially considering all possible network configurations in section 3. A mixed-integer nonlinear programming (MINLP) formulation follows for modeling the synthesis work and storage policy in section 4. Numerical examples are thereafter illustrated for demonstrating the proposed design procedure in section 5 . Finally, a conclusion is made for summary.

\section{Problem Statement}

The continuous MEN synthesis problem following a nonconsecutive process, as addressed in this paper, can be stated as follows. Given are a set of upstream plants $a \in \mathrm{A}$ which are operated alternatively to provide semiconsecutive process streams for subsequent treatment; a set of intermittent or continuous rich process streams $i \in \mathrm{RP}$ in which some transferable components $n \in \mathrm{TC}$ are to be removed away; a set of intermittent or continuous lean mass-separating agents (MSAs) $j \in \mathrm{LP}$ which are used for removing those transferable components in rich streams; a set of periods $p \in \mathrm{TP}$ defining the 


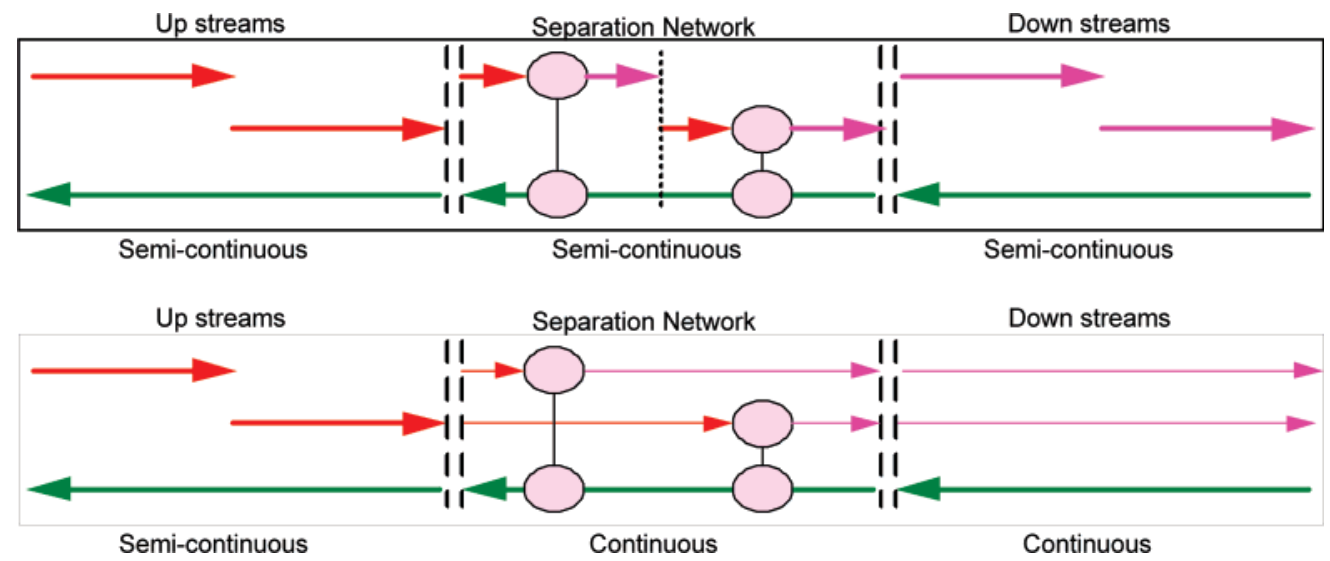

Figure 1. Operating types for mass-exchange networks (MENs), following a semiconsecutive process: (a) intermittent MEN mode and (b) continuous MEN mode.

starting and ending times of various streams; and sets of gasphase streams $g \in \mathrm{NCR}^{\text {(gas) }} \cup \mathrm{NCL}^{\text {(gas) }}$ and liquid-phase streams $l \in \mathrm{NCR}^{(\text {liq) }} \cup \mathrm{NCL}^{\text {(liq) }}$ appeared in the rich and/or lean flows.

Also specified parameters are the period-dependent flow rates of available rich process streams from plants $G_{a i p}$, and maximum available rates for lean process streams $L_{a j \mathrm{p}}^{(\mathrm{up})}$; input, output, and upper compositions for rich streams $\left(Y_{i}^{n(\text { in })}, Y_{i}^{n \text { (out) }}\right.$, and $Y_{i}^{n \text { (up) }}$, respectively) and for lean streams $\left(X_{j}^{n \text { (in) }}, X_{j}^{n \text { (out) }}\right.$, and $X_{j}^{n \text { (up) }}$, respectively). Also given are linear equilibrium relations for the distribution of transferable components between rich and lean flows, $y_{i}^{n^{*}}=m_{i j}^{n} x_{j}^{n}+b_{i j}^{n}$, where $y_{i}^{n^{*}}$ and $x_{j}^{n}$ are equilibrium compositions of component $n$ in the $i$ th rich and $j$ th lean streams, respectively, $m_{i j}^{n}$ and $b_{i j}^{n}$ are associated equilibrium constants, and $\epsilon_{i j}^{n}$ is a minimum composition approach. The objective then is to determine the time-independent MEN with associated storing strategies for periodically dependent inlet flows that target the total annual cost (TAC).

The solution about the continuously operated MEN is provided in the following: the stream matches and the number of units, the targeting MSAs and those of mass flow rates, the network configuration and flows for all branches, and the massexchange rates and operating compositions of each massexchanger unit. In addition, the solution about the storing policy of time-dependent streams is provided in the following: the inlet and outlet flow rates of vessels/tanks at different periods, the existence of storage vessels/tanks and their remained quantities at each time point, and the sizes of vessels/tanks and compressors. The overall network with storage vessels/tanks then is constructed based on this information.

Several assumptions are made to simplify the continuous MEN synthesis problem, including the following: ${ }^{1,10}$

(1) The concentration of each stream remains constant.

(2) The equilibrium of each transferred component is not dependent on the other components.

(3) The mass-exchange units are counter-current type.

(4) Mass exchange between any two rich streams and between any two lean streams is not allowed.

(5) Heat integration between streams is not considered.

(6) The network operates at constant pressure.

In addition, we assume that this does not pose a problem in the COG case study.

\section{The Periodically Partitioned Stagewise Superstructure for MEN Synthesis}

A periodically partitioned approach is proposed to overcome the different starting and ending times of various inlet streams in a semiconsecutive process. ${ }^{10}$ For any period $p \in \mathrm{TP}$, Figure 2 illustrates a typical two-stage superstructure with two upstream plants, two rich streams $\left(R_{1}, R_{2}\right)$ and two lean streams $\left(S_{1}, S_{2}\right)$, and the associated storage tanks for inlet streams. The two stages are represented by eight mass-exchange units, with four possible matches in each stage and variable compositions between each stage. The number of stages required to model the mass integration is seldom greater than either the maximal number of rich streams $N_{R}$, or the maximal number of lean streams $N_{L}$, at all periods. ${ }^{8}$ Thus, the number of stages is typically fixed at $N_{S}=\max \left\{N_{R}, N_{L}\right\}$. However, one additional stage is sometimes recommended to search for potential better networks. Note that, in all periods $p \in \mathrm{TP}$, the MEN is permanently operated in continuous mode, although the input streams may be not available in some intervals. Therefore, part of the inlet streams must be stored temporarily in storage vessels/tanks in surplus periods, and the accumulated material can be released late during deficit intervals for subsequent treatment.

It is also found that the inlet rates of rich/lean streams flow into the continuous MEN ( $G E_{i}^{\prime}$ s and $L E_{j}^{\prime}$ s) are the key variables that connect the network operation and the streams storage. Therefore, the continuous MEN synthesis problem can be divided into two serial phases: the first phase concerns the synthesis of the continuously operated network configuration, and the second phase involves the period-dependent storing/ releasing strategy for inlet streams, which includes storing during surplus periods and releasing in deficit intervals. Note that the values of connecting variables are solely determined for providing the optimal network configuration in design phase I, and design phase II takes these values as given parameters in resolving the streams storage. Although one can simultaneously determine the optimal continuous MEN with the associated streams storage policy by minimizing the overall TAC, the sequential design steps will be adopted in the article for increasing the possibility of numerical convergence. Detailed model formulations are given in the next section.

\section{Model Formulation for a Continuous Mass-Exchange Network (MEN) Following a Nonconsecutive Process}

As mentioned previously, the synthesis of a continuous MEN following a semiconsecutive process consists of two design phases in the sequential approach: the synthesis of a continuously operated MEN (Design Phase I) and a storing policy for handling intermittent inlet streams (Design Phase II). The twophase design problem is investigated sequentially in the following discussion. 


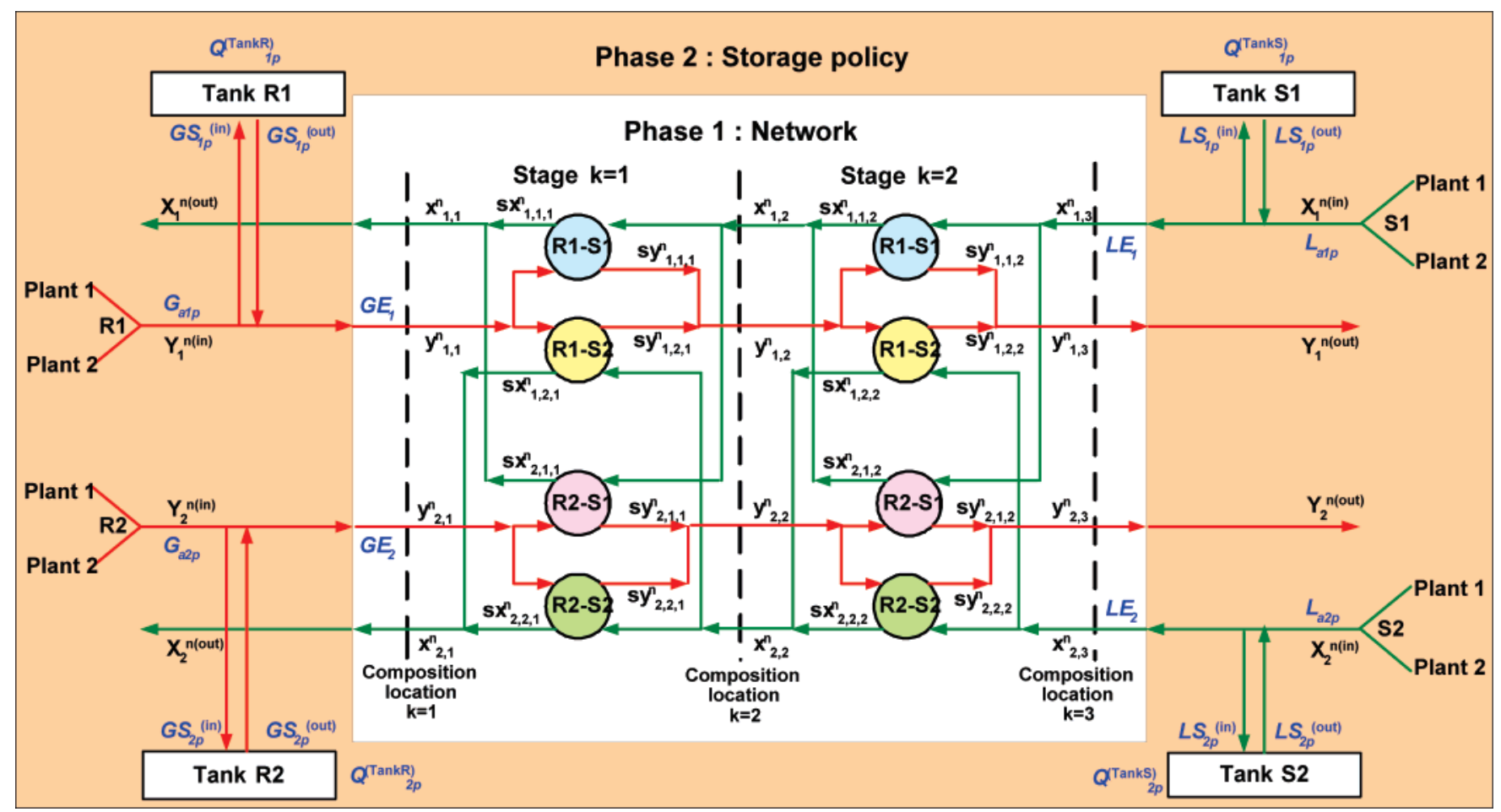

Figure 2. Schematic of a two-stage superstructure with two rich (or two lean) streams and associated storage vessels/tanks.

4.1. Modeling for Continuous MEN (Design Phase I). To model the generic stagewise superstructure for the synthesis of a continuous MEN, the indices, sets, parameters, and variables are given below.

\section{Indices}

$a=$ index for plants

$i=$ index for rich process streams and storage tanks

$j=$ index for lean process streams

$k=$ index for superstructure stages, $k=1, \ldots, N_{S}$; and composition locations, $k=1, \ldots, N_{S}+1$

$n=$ index for transferrable components

\section{Sets}

$\mathrm{A}=\left\{a \mid a\right.$ is a plant, $\left.i=1, \ldots, N_{A}\right\}$

$\mathrm{LP}=\{j \mid j$ is a lean (process) stream or MSA, $\left.j=1, \ldots, N_{L}\right\}$

$\mathrm{RP}=\left\{i \mid i\right.$ is a rich process stream, $\left.i=1, \ldots, N_{R}\right\}$

$\mathrm{ST}=\left\{k \mid k\right.$ is a stage in the superstructure, $\left.k=1, \ldots, N_{S}\right\}$

$\mathrm{TC}=\left\{n \mid n\right.$ is a transferrable component, $\left.n=1, \ldots, N_{C}\right\}$

\section{Parameters}

$A C_{i j}^{(\mathrm{tr})}=$ per stage annual cost of tray column for rich stream $i$ and lean stream $j$

$A C_{i j}^{(\mathrm{h})}=$ per high annual cost of packed column for rich stream $i$ and lean stream $j$ $b_{i j}^{n}=$ intercept of the equilibrium line for component $n$ where rich stream $i$ and lean stream $j$ match

$C_{j}=$ cost per kilogram of lean stream $j$

$G_{\text {aip }}=$ mass flow rate of rich stream $i$ at period $p$ in plant $a(\mathrm{~kg} / \mathrm{h})$

$G E_{i}=$ mass flow rate of rich stream $i$ flows into stage $(\mathrm{kg} / \mathrm{h})$

$K_{y} a=$ overall mass-transfer coefficient

$L_{a j p}^{(\text {up) }}=$ upper bound on mass flow rate of lean stream $j$ at period $p$ in plant $a(\mathrm{~kg} / \mathrm{h})$

$L E_{j}^{(\text {up) }}=$ upper bound on mass flow rate of lean stream $j$ flows into stage $(\mathrm{kg} / \mathrm{h})$

$m_{i j}^{n}=$ slope of the equilibrium line for component $n$ where rich stream $i$ and lean stream $j$ match

$\bar{M}, \underline{M}=$ a large/small positive number

$N_{\text {B }}=$ number of batch operation time per year

$S=$ cross-sectional area of an exchange unit

$t_{p}=$ elapsed time of period $p(\mathrm{~h})$

$X_{j}^{n(\text { in) }}=$ inlet composition of lean stream $j$ for component $n$

$X_{j}^{n \text { (out) }}=$ outlet composition of lean stream $j$ for component $n$

$X_{j}^{n(\text { up })}=$ upper composition of lean stream $j$ for component $n$ 


$$
\begin{aligned}
Y_{i}^{n(\text { in })}= & \text { inlet composition of rich stream } i \text { for } \\
& \text { component } n \\
Y_{i}^{n(\text { out })}= & \text { outlet composition of rich stream } i \text { for } \\
& \text { component } n \\
Y_{i}^{n(\text { up })}= & \text { upper composition of rich stream } i \text { for } \\
& \text { component } n \\
\sigma= & \text { a small positive number } \\
\epsilon_{i j}^{n}= & \text { minimum composition difference of composition } \\
& n \text { between rich stream } i \text { and lean stream } j \\
\Gamma= & \text { a large positive upper bound } \\
\text { MEU }= & \text { maximum total number of mass-exchange units }
\end{aligned}
$$

\section{Positive and Binary Variables}

$g_{i j k}=$ mass flow rate of rich stream $i$ that is connected to lean stream $j$ in stage $k(\mathrm{~kg} / \mathrm{h})$

$H_{i j k}=$ height of the packed column of match $(i, j)$ in stage $k$

$l_{i j k}=$ mass flow rate of lean stream $j$ that is connected to rich stream $i$ in stage $k(\mathrm{~kg} / \mathrm{h})$

$L E_{j}=$ mass flow rate of lean stream $j$ flows into stage $(\mathrm{kg} / \mathrm{h})$

$M_{i j k}^{n}=$ mass load per hour exchanged for component $n$ between rich stream $i$ and lean stream $j$ in stage $k(\mathrm{~kg} / \mathrm{h})$

$N_{i j k}=$ integer number of trays in the tray column of match $(i, j)$ in stage $k$

$s x_{i j k}^{n}=$ composition of component $n$ for the part of lean stream $j$ that is connected to rich stream $i$ in the rich end of an exchanger in stage $k$

$s y_{i j k}^{n}=$ composition of component $n$ for the part of rich stream $i$ that is connected to lean stream $j$ in the rich end of an exchanger in stage $k$

$x_{j k}^{n}=$ composition of component $n$ in lean stream $j$ at rich end of stage $k$

$y_{i k}^{n}=$ composition of component $n$ in rich stream $i$ at rich end of stage $k$

$z_{i j k}=\in\{0,1\}$, where 1 denotes the existence of match $(i, j)$ in stage $k$

Note that the inlet stream flow rates, $G E_{i}$ and $L E_{j}^{(\text {up })}$, are given parameters in the phase of continuous network synthesis, and they can be calculated from eq 1 for supplying steady rates for the MEN.

$$
G E_{i}=\frac{\sum_{\forall a \in \mathrm{A}} \sum_{\forall p \in \mathrm{TP}} G_{\text {aip }} t_{p}}{\sum_{\forall p \in \mathrm{TP}} t_{p}} \forall i \in \mathrm{RP}
$$

$$
L E_{j}^{\text {(up) }}=\frac{\sum_{\forall a \in \mathrm{A}} \sum_{\forall p \in \mathrm{TP}} L_{a j p}^{(\mathrm{up})} t_{p}}{\sum_{\forall p \in \mathrm{TP}} t_{p}} \forall j \in \mathrm{LP}
$$

With these definitions, we can model the stagewise superstructure for synthesizing the continuously operated MEN following a semiconsecutive process, as follows. ${ }^{8}$

4.1.1. Overall Mass Balances for Transferable Components over the Entire Network. An overall mass balance is formulated to ensure sufficient exchange of all transferred components for all rich and lean streams:

$$
\begin{aligned}
& \left(Y_{i}^{n(\text { in })}-Y_{i}^{n(\text { out })}\right) G E_{i}=\sum_{\forall k \in \mathrm{ST}} \sum_{\forall j \in \mathrm{LP}} M_{i j k}^{n} \\
& \forall i \in \mathrm{RP}, n \in \mathrm{TC} \\
& \left(X_{j}^{n(\text { out })}-X_{j}^{n(\text { in })}\right) L E_{j}=\sum_{\forall k \in \mathrm{ST}} \sum_{\forall i \in \mathrm{RP}} M_{i j k}^{n} \\
& \forall j \in \mathrm{LP}, n \in \mathrm{TC}
\end{aligned}
$$

The first constraint specifies that the overall transferrable requirement for component $n$ of each rich stream $i$ must be equal to the sum of exchange loads for the component, which are exchanged with other lean process streams or external MSAs at all potential mass-exchange units. The second constraint reveals the similar relation for component $n$ in lean stream $j$, should it be available.

4.1.2. Mass Balances for Transferable Components in Each Stage. A mass balance of each stream in each stage is used to determine the composition of each transferrable component. For a superstructure with $N_{S}$ stages, $N_{S}+1$ levels of composition are involved. Note that the index $k$ is used to represent both the stage and the composition location in the superstructure. For each stage $k$, the composition at location $k$ of each stream is higher than the composition at location $k+$ 1. The component and the mass balance for each stream in each stage can be stated as follows:

$$
\begin{aligned}
&\left(y_{i k}^{n}-y_{i, k+1}^{n}\right) G E_{i}=\sum_{\forall j \in \mathrm{LP}} M_{i j k}^{n} \\
&\left(x_{j k}^{n}-x_{j, k+1}^{n}\right) L E_{i}=\sum_{\forall i \in \mathrm{RP}} M_{i j k}^{n} \forall i \in \mathrm{RP}, k \in \mathrm{ST}, n \in \mathrm{TC} \\
& \forall j \in \mathrm{LP}, k \in \mathrm{ST}, n \in \mathrm{TC}
\end{aligned}
$$

4.1.3. Mass Balances for Transferable Components in Each Exchange Unit. A component mass balance is needed for each local exchange unit. The inlet composition of each unit is the same as the inlet composition at each stage, because we assume that the action of splitting does not affect the composition of each stream. New composition variables, $s y_{i j k}^{n}$ and $s x_{i j k}^{n}$, are defined to express outlet compositions of units, as illustrated in Figure 2. The unit mass balance is stated as follows:

$$
\begin{gathered}
\left(y_{i k}^{n}-s y_{i j k}^{n}\right) g_{i j k}=M_{i j k}^{n} \\
\left(s x_{i j k}^{n}-x_{j, k+1}^{n}\right) l_{i j k}=M_{i j k}^{n} \\
\forall i \in \mathrm{RP}, j \in \mathrm{LP}, k \in \mathrm{ST}, n \in \mathrm{TC}, j \in \mathrm{LP}, k \in \mathrm{ST}, n \in \mathrm{TC}
\end{gathered}
$$

Notice that the balance equations around all mixers from split streams are not necessary, because these equations are redundant to eqs 3 and 4. 
4.1.4. Mass Rate Balances in Each Stage. For each stage, each rich stream $i$ splits into $N_{L}$ streams to match with $N_{L}$ lean streams, respectively. New variables, $g_{i j k}$ and $l_{i j k}$, are used to represent the split mass rates for rich stream $i$ and lean stream $j$ at stage $k$.

$$
\begin{aligned}
G E_{i}=\sum_{\forall j \in \mathrm{LP}} g_{i j k} & \forall i \in \mathrm{RP}, k \in \mathrm{ST} \\
L E_{j}=\sum_{\forall i \in \mathrm{RP}} l_{i j k} & \forall j \in \mathrm{LP}, k \in \mathrm{ST}
\end{aligned}
$$

When the unit does not exist, the passing flow rate must be zero, as follows:

$$
\begin{gathered}
g_{i j k}-G E_{i} z_{i j k} \leq 0 \quad \forall i \in \mathrm{RP}, j \in \mathrm{LP}, k \in \mathrm{ST} \\
l_{i j k}-L E_{j} z_{i j k} \leq 0
\end{gathered}
$$

However, eq 6 will clash with eq 5 when all of the units of a specific stream match with any lean streams for one stage $\left(\sum_{\forall j \in L P} z_{i j k}=0\right)$. To avoid having no solutions, eq 6 is multiplied by $\sum_{\forall j \in L P} z_{i j k}$ as follows:

$$
\begin{array}{ll}
\left(\sum_{\forall j \in \mathrm{LP}} z_{i j k}\right)\left(g_{i j k}-G E_{i} z_{i j k}\right) \leq 0 & \forall i \in \mathrm{RP}, j \in \mathrm{LP}, k \in \mathrm{ST} \\
\left(\sum_{\forall j \in \mathrm{LP}} z_{i j k}\right)\left(l_{i j k}-L E_{j} z_{i j k}\right) \leq 0 & \forall i \in \mathrm{RP}, j \in \mathrm{LP}, k \in \mathrm{ST}
\end{array}
$$

4.1.5. Assignment of Superstructure Inlet/Outlet Compositions. The given inlet/outlet compositions of rich and lean process streams are assigned as the inlet/outlet compositions to the superstructure. For rich streams, the inlet of the superstructure corresponds to composition location $k=1$; the outlet of the superstructure corresponds to composition location $k=N_{S}$ +1 . For lean streams, the inlet of the superstructure corresponds to location $k=N_{S}+1$; the outlet of the superstructure corresponds to composition location $k=1$.

$$
\begin{array}{ll}
Y_{i}^{n(\mathrm{in})}=y_{i 1}^{n}, \quad Y_{i}^{n(\mathrm{out})}=y_{i, N_{S}+1}^{n} & \forall i \in \mathrm{RP}, n \in \mathrm{TC} \\
Y_{j}^{n(\mathrm{in})}=x_{j, N_{S}+1}^{n}, \quad X_{j}^{n(\text { out })}=x_{j 1}^{n} & \forall j \in \mathrm{LP}, n \in \mathrm{TC}
\end{array}
$$

4.1.6. Bounds on Variables. Bounds for available masses of lean process streams flowing through the network should be set. Bounds for final compositions of rich and lean streams comply with the rules of environmental protection.

$$
\begin{aligned}
L E_{j} \leq L E_{j}^{\text {(up) }} & X_{j}^{n(\text { out })} \leq X_{j}^{n(\text { up })} \quad \forall j \in \mathrm{LP}, n \in \mathrm{TC} \\
& Y_{i}^{n(\text { out })} \leq Y_{i}^{n \text { (up) }} \quad \forall i \in \mathrm{RP}, n \in \mathrm{TC}
\end{aligned}
$$

4.1.7. Feasibility of the Transferable Components. Constraints are also needed to guarantee a monotonic decrease of all compositions at successive stages, such as

$$
\begin{array}{ll}
y_{i k}^{n} \geq y_{i, k+1}^{n} & \forall i \in \mathrm{RP}, k \in \mathrm{ST}, n \in \mathrm{TC} \\
x_{j k}^{n} \geq x_{j, k+1}^{n} & \forall j \in \mathrm{LP}, k \in \mathrm{ST}, n \in \mathrm{TC}
\end{array}
$$

For an existing unit, the output component of the rich streams cannot be greater than the input component, and output component of the lean streams cannot be below the input component. When the unit does not exist, the output component of the rich and lean streams must be equal to the input component.

$$
\begin{aligned}
& s y_{i j k}^{n} \leq y_{i k}^{n} \leq s y_{i j k}^{n}+ Y_{i}^{n(\text { in })} z_{i j k} \\
& \forall i \in \mathrm{RP}, j \in \mathrm{LP}, k \in \mathrm{ST}, n \in \mathrm{TC} \\
& x_{j, k+1}^{n} \leq s x_{i j k}^{n} \leq x_{j, k+1}^{n}+X_{j}^{n(\text { out })} z_{i j k} \\
& \forall i \in \mathrm{RP}, j \in \mathrm{LP}, k \in \mathrm{ST}, n \in \mathrm{TC}
\end{aligned}
$$

4.1.8. Feasibility Constraints of the Equilibrium Relationships. Linear equilibrium relations are adopted here for distribution of transferable component $n$ between rich $i$ and lean $j$ streams, $y_{i}^{n^{*}}=m_{i j}^{n} x_{j}^{n}+b_{i j}^{n}$. The feasibility constraints of the equilibrium relationships ensure positive driving forces, $y_{i k}^{n}-s$ $y_{i j k}^{n^{*}}$ and $s y_{i j, k+1}-y_{i, k+1}^{n^{*}}$, for the potential $(i, j)$ match in stage $k$ at the rich and lean sides, respectively. Binary variables are used for these constraints to ensure that only the non-negative driving forces exist for existing matches where the associated binary variables all are equal to 1 . If a match does not occur, the associated binary variable equals zero and the large positive upper bound $\Gamma$ can deem the equation redundant. In these equations, a streams- and component-dependent minimum composition approach $\epsilon_{i j}^{n}$ is also chosen, so that feasible mass transfer in a finite number of equilibrium stages or finite area can be achieved in each transfer unit. ${ }^{7}$

$$
\begin{aligned}
y_{i k}^{n}-\left[m_{i j}^{n}\left(s x_{i j k}^{n}+\epsilon_{i j}^{n}\right)\right. & \left.+b_{i j}^{n}\right]+\Gamma\left(1-z_{i j k}\right) \geq 0 \\
& \forall i \in \mathrm{RP}, j \in \mathrm{LP}, k \in \mathrm{ST}, n \in \mathrm{TC} \\
s y_{i j, k}^{n}-\left[m _ { i j } ^ { n } \left(x_{j, k+1}^{n}+\right.\right. & \left.\left.\epsilon_{i j}^{n}\right)+b_{i j}^{n}\right]+\Gamma\left(1-z_{i j k}\right) \geq 0 \\
& \forall i \in \mathrm{RP}, j \in \mathrm{LP}, k \in \mathrm{ST}, n \in \mathrm{TC}
\end{aligned}
$$

4.1.9. Logical Constraints. Logical constraints and binary variables $\left(z_{i j k}\right)$ are used to determine the existence or absence of process matches $(i, j)$ in stage $k$. An integer value of 1 for binary variable $z_{i j k}$ designates that a match between rich stream $i$ and lean stream $j$ in stage $k$ is present in the optimal network. The constraints are as follows:

$$
\underline{M} z_{i j k} \leq \sum_{\forall n \in \mathrm{TC}} M_{i j k}^{n} \leq \bar{M} z_{i j k} \quad \forall i \in \mathrm{RP}, j \in \mathrm{LP}, k \in \mathrm{ST}
$$

where $\underline{M}$ and $\bar{M}$ are positive lower and upper bounds for the exchangers' load.

4.1.10. Optional Constraints. Some additional constraints, such as no stream splits, forbidden matches, and required and restricted matches, can be easily included in this model. For example, the stream splitting can be prevented by constraining the number of matches for split streams in each stage, such as

$$
\begin{array}{ll}
\sum_{\forall i \in \mathrm{RP}} z_{i j k} \leq 1 & \forall j \in \mathrm{LP}, k \in \mathrm{ST} \\
\sum_{\forall j \in \mathrm{LP}} z_{i j k} \leq 1 & \forall i \in \mathrm{RP}, k \in \mathrm{ST}
\end{array}
$$

The maximum total number of mass-exchange units can be limited by adding an upper bound for selected exchangers (MEU) in the following constraint:

$$
\sum_{\forall i \in \mathrm{RP}} \sum_{\forall j \in \mathrm{LP}} \sum_{\forall k \in \mathrm{ST}} z_{i j k} \leq \mathrm{MEU}
$$

Other restrictions also can be considered by assigning suitable values for specific integer variables. For example, if the match between rich stream $i=1$ and lean stream $j=2$ is not allowable, then one can assign $z_{12 k}=0, \forall k \in \mathrm{ST}$. 
4.1.11. Sizing Equations for Mass-Transfer Units. Massexchange units can be classified into two main categories: the stagewise exchangers and the continuous-contact exchangers. ${ }^{12}$ The most common types of stagewise exchangers are tray or plate columns. When mass exchange happens in a tray column, the number of required stages can be determined from the Kremser equation. The traditional form for the Kremser equation can be expressed as follows, if the operating and equilibrium lines are both straight: ${ }^{13-15}$

$$
N_{i j k}^{n}=\frac{\ln \left[\left(\frac{y_{i k}^{n}-y_{i j, k+1}^{n^{*}}}{s y_{i j k}^{n}-y_{i j, k+1}^{n^{*}}}\right)\left(1-\frac{m_{i j}^{n} g_{i j k}}{l_{i j k}}\right)\right]}{\ln \left[\frac{l_{i j k}}{m_{i j}^{n} g_{i j k}}\right]}
$$

$\forall i \in \mathrm{RP}, j \in \mathrm{LP}, k \in \mathrm{ST}, n \in \mathrm{TC}$

$$
\left(\text { for } \frac{l_{i j k}}{m_{i j}^{n} g_{i j k}} \neq 1\right)
$$

$N_{i j k}^{n}=\frac{y_{i k}^{n}-s y_{i j k}^{n}}{s y_{i j k}^{n}-y_{i j, k+1}^{n^{*}}}$

$$
\forall i \in \mathrm{RP}, j \in \mathrm{LP}, k \in \mathrm{ST}, n \in \mathrm{TC} \quad\left(\text { for } \frac{l_{i j k}}{m_{i j}^{n} g_{i j k}}=1\right)
$$

where $y_{i j, k+1}^{n^{*}}$ and $s y_{i j k}^{n^{*}}$ are equilibrium compositions $\left(y_{i j, k+1}^{n^{*}}=\right.$ $\left.m_{i j}^{n} x_{\mathrm{j}, k+1}^{n}+b_{i j}^{n}, s y_{i j k}^{n^{*}}=m_{i j}^{n} s x_{i j k}^{n}+b_{i j}^{n}\right)$. The linear equilibrium relation and the material balance equation can be further substituted into eqs 16 to give the following alternative form of the Kremser equation: ${ }^{14}$

$$
\begin{aligned}
N_{i j k}^{n}= & \frac{\operatorname{LMCD}\left[y_{i k}^{n}-s y_{i j k}^{n} s y_{i j k}^{n^{*}}-y_{i j, k+1}^{n^{*}}\right]}{\operatorname{LMCD}\left[y_{i k}^{n}-s y_{i j k}^{n^{*}}, s y_{i j k}^{n}-y_{i j, k+1}^{n^{*}}\right]} \\
& \forall i \in \mathrm{RP}, j \in \mathrm{LP}, k \in \mathrm{ST}, n \in \mathrm{TC} \quad\left(\text { for } \frac{l_{i j k}}{m_{i j}^{n} g_{i j k}} \neq 1\right)
\end{aligned}
$$

$$
\begin{aligned}
N_{i j k}^{n}= & \frac{y_{i k}^{n}-s y_{i j k}^{n}}{y_{i k}^{n}-s y_{i j k}^{n^{*}}}=\frac{y_{i k}^{n}-s y_{i j k}^{n}}{s y_{i j k}^{n}-y_{i j, k+1}^{n^{*}}} \\
& \forall i \in \mathrm{RP}, j \in \mathrm{LP}, k \in \mathrm{ST}, n \in \mathrm{TC} \quad\left(\text { for } \frac{l_{i j k}}{m_{i j}^{n} g_{i j k}}=1\right)
\end{aligned}
$$

where LMCD represents the logarithmic mean (log-mean) of the two concentrations given in the brackets. Notably, the logmean Kremser representation for the case of $l_{i j k} /\left(m_{i j}^{n} g_{i j k}\right) \neq 1$ will be equivalent to the second one for the $l_{i j k}\left(m_{i j j}^{n} g_{i j k}\right)=1$ case. However, eqs 17 will still lead to numerical difficulties for some zero or negative values in the log-mean. Thus, an approximation for the composition difference term is required to avoid numerical problems when the approach compositions of both sides of the mass-exchange unit are equal. ${ }^{14,16,17}$ There are many approximations for the log-mean term. Here, Chen's approximation ${ }^{14,18}$ is used:

$$
\begin{aligned}
\operatorname{LMCD}\left[y_{i k}^{n}-s y_{i j k}^{n}, s y_{i j k}^{n^{*}}-y_{i j, k+1}^{n^{*}}\right] & = \\
& \frac{\left(y_{i k}^{n}-s y_{i j k}^{n}\right)-\left(s y_{i j k}^{n^{*}}-y_{i j, k+1}^{n^{*}}\right)}{\ln \left[\frac{y_{i k}^{n}-s y_{i j k}^{n}}{s y_{i j k}^{n^{*}}-y_{i j, k+1}^{n^{*}}}\right]}
\end{aligned}
$$

$$
\begin{aligned}
& \simeq\left[\frac{\left(y_{i k}^{n}-s y_{i j k}^{n}\right)^{0.3275}+\left(s y_{i j k}^{n^{*}}-y_{i j, k+1}^{n^{*}}\right)^{0.3275}}{2}\right]^{1 / 0.3275} \\
& \forall i \in \mathrm{RP}, j \in \mathrm{LP}, k \in \mathrm{ST}, n \in \mathrm{TC}
\end{aligned}
$$

Substituting eq 18 into eqs 17 transforms eqs 17 as follows:

$$
\begin{array}{r}
N_{i j k}^{n} \cong\left[\frac{\left(y_{i k}^{n}-s y_{i j k}^{n}\right)^{0.03275}+\left(s y_{i j k}^{n^{*}}-y_{i, k+1}^{n^{*}}\right)^{0.03275}}{\left(y_{i j k}^{n}-s y_{i j k}^{n^{*}}\right)^{0.03275}+\left(s y_{i j k}^{n}-y_{i j, k+1}^{n^{*}}\right)^{0.03275}}\right]^{1 / 0.03275} \\
\forall i \in \mathrm{RP}, j \in \mathrm{LP}, k \in \mathrm{ST}, n \in \mathrm{TC}
\end{array}
$$

Notably, the final stage number is chosen as the maximal stages for all components if the tray column is selected. Moreover, we use the variable $z_{i j k}$ to ensure that the stage is zero when the unit is nonexistent.

$$
\begin{aligned}
& N_{i j k} \geq z_{i j k} \\
& {\left[\frac{\left(y_{i k}^{n}-s y_{i j k}^{n}+\sigma\right)^{0.03275}+\left(s y_{i j k}^{n^{*}}-y_{i, k+1}^{n^{*}}+\sigma\right)^{0.03275}}{\left(y_{i j k}^{n}-s y_{i j k}^{n^{*}}\right)^{0.03275}+\left(s y_{i j k}^{n}-y_{i j, k+1}^{n^{*}}\right)^{0.03275}}\right]^{1 / 0.03275}} \\
& \forall i \in \mathrm{RP}, j \in \mathrm{LP}, k \in \mathrm{ST}, n \in \mathrm{TC}
\end{aligned}
$$

The aforementioned smoothing equation has been incorporated into some NLP solvers, such as the SNOPT, using optimization solution tools, such as the General Algebraic Modeling System $\left(\mathrm{GAMS}^{19}\right)$. When absorption or stripping occurs, a continuous-contact packed tower is suggested for mass exchange. The required packed height for an $(i, j)$ match in stage $k$, for the separation of component $n\left(H_{i j k}^{n}\right)$, is characterized by several imaginary transfer units $\left(\mathrm{NTU}_{i j k}^{n}\right)$ and the overall height of a transfer unit $\left(\mathrm{HTU}_{i j k}^{n}\right)$. 5,15 Therein, calculations are based on the conditions in the rich stream. The overall packed height is given by the following equation, where the log-mean calculation is also made by applying Chen's approximation and the smooth approximation method.

$$
\begin{aligned}
& \mathrm{HTU}_{i j k}^{n}=\frac{g_{i j k}}{K_{y}^{n} a S} \quad \forall i \in \mathrm{RP}, j \in \mathrm{LP}, k \in \mathrm{ST}, n \in \mathrm{TC} \\
& \mathrm{NTU}_{i j k}^{n}=\frac{y_{i k}^{n}-s y_{i j k}^{n}}{\operatorname{LMCD}\left[y_{i k}^{n}-s y_{i j k}^{n^{*}}, s y_{i j k}^{n}-y_{i j, k+1}^{n^{*}}\right]} \\
& H_{i j k}^{n}=\mathrm{HTU}_{i j k}^{n} \times \mathrm{NTU}_{i j k}^{n} \\
&=\frac{M_{i j k}^{n}}{K_{y}^{n} a S} \times \frac{1}{\mathrm{LMCD}\left[y_{i k}^{n}-s y_{i j k}^{n^{*}}, s y_{i j k}^{n}-y_{i j, k+1}^{n^{*}}\right]} \\
&=\frac{M_{i j k}^{n}}{K_{y}^{n} a S}\left[\frac{\left(y_{i k}^{n}-s y_{i j k}^{n^{*}}\right)^{0.3275}+\left(s y_{i j k}^{n}-y_{i j, k+1}^{n^{*}}\right)^{0.3275}}{2}\right]^{-1 / 0.3275} \\
& \forall i \in \mathrm{RP}, j \in \mathrm{LP}, k \in \mathrm{ST}, n \in \mathrm{TC}
\end{aligned}
$$

In the same way, the largest exchanger height is chosen as the maximal stages for all components, if the plate column is selected. In addition, we use the variable $z_{i j k}$ to ensure that the stage is zero when the unit is nonexistent.

$$
\begin{aligned}
& H_{i j k} \geq z_{i j k} \frac{M_{i j k}^{n}}{K_{y}^{n} a S} \\
& {\left[\frac{\left(y_{i k}^{n}-s y_{i j k}^{n^{*}}\right)^{0.3275}+\left(s y_{i j k}^{n}-y_{i j, k+1}^{n *}\right)^{0.3275}}{2}\right]^{-1 / 0.3275} } \\
& \forall i \in \mathrm{RP}, j \in \mathrm{LP}, k \in \mathrm{ST}, n \in \mathrm{TC}
\end{aligned}
$$


4.2. Modeling for Storing Policy (Design Phase II). To model the storing policy for semiconsecutive inlet streams, additional indices, sets, parameters, and variables are given below. Note that the mass flow rates of the lean streams $\left(L E_{j} \mathrm{~s}\right)$ become parameters in the streams storage phase, which are supplied from the design result of the previous MEN synthesis phase.

1. Indices (additional). With the exception of those indices in the MEN synthesis phase, additional indices are required to model the mass storage phase:

$$
\begin{aligned}
& g=\text { index for noncontinuous gas streams, } \\
& \text { pressure vessels, and compressors } \\
& l=\text { index for noncontinuous liquid streams and } \\
& \text { liquid vessels } \\
& s=\text { index for pressure stages }
\end{aligned}
$$

2. Sets

$$
\begin{aligned}
\mathrm{CL}= & \{j \mid j \text { is a continuous lean (process) stream or } \\
& \mathrm{MSA}\} \\
\mathrm{CR}= & \{i \mid i \text { is a continuous rich process stream }\} \\
\mathrm{NCL}= & \{j \mid j \text { is a noncontinuous lean (process) stream } \\
& \text { or MSA }\} \\
\mathrm{NCL}^{(\text {gas })}= & \{j \mid j \text { is a gas-phase noncontinuous lean (process) } \\
& \text { stream or MSA }\} \\
\mathrm{NCL}^{(\text {liq })}= & \{j \mid j \text { is a liquid-phase noncontinuous lean } \\
& (\text { process) stream or MSA }\} \\
\mathrm{NCR}= & \{i \mid i \text { is a noncontinuous rich process stream }\} \\
\mathrm{NCR}^{(\mathrm{gas})}= & \{i \mid i \text { is a gas-phase noncontinuous rich process } \\
& \text { stream }\} \\
\mathrm{NCR}^{(\text {liq })=} & \{i \mid i \text { is a liquid-phase noncontinuous rich } \\
& \text { process stream }\} \\
\mathrm{PSS}= & \{s \mid s \text { is a pressure stage }\}
\end{aligned}
$$

\section{Parameters}

Chp $=$ cost per horsepower

$F \mathrm{c}_{g}=$ correction factor for compressor of gas stream $g$

$F \mathrm{~m}_{g}=$ material correction factor for pressure vessel $g$

$F \mathrm{~m}_{l}=$ material correction factor for liquid vessel $l$

$F_{s}=$ correction factor for compressor of pressure stage $s$

$G_{\text {aip }}=$ mass flow rate of rich stream $i$ at period $p$ in plant $a(\mathrm{~kg} / \mathrm{h})$

$G E_{i}=$ mass flow rate of rich stream $i$ flows into stage $(\mathrm{kg} / \mathrm{h})$

$L_{a j p}^{\text {(up) }}=$ upper bound on mass flow rate of lean stream $j$ at period $p$ in plant $a(\mathrm{~kg} / \mathrm{h})$

$L E_{j}=$ mass flow rate of lean stream $j$ flows into stage $(\mathrm{kg} / \mathrm{h})$

$N_{\mathrm{B}}=$ number of batch operation time per year

$$
\begin{aligned}
P_{g}^{(\text {in })}= & \text { input pressure of compressor for gas stream } \\
& g(\text { atm }) \\
P_{g}^{(\text {lo })}= & \text { lower pressure of compressor for gas stream } \\
& g(\text { atm }) \\
P_{g}^{\text {up })}= & \text { upper pressure of compressor for gas stream } \\
& g(\text { atm }) \\
P_{s}= & \text { pressure of stage } s(\text { atm }) \\
t_{p}= & \text { elapse time of period } p(\mathrm{~h}) \\
\bar{U}= & \text { upper bound } \\
\underline{U}= & \text { lower bound } \\
\rho_{g}^{(\text {in })}= & \text { input density of gas stream } g\left(\mathrm{~kg} / \mathrm{m}^{3}\right) \\
\rho_{l}^{(\text {up })}= & \text { input density of liquid stream } l\left(\mathrm{~kg} / \mathrm{m}^{3}\right) \\
\gamma= & \text { value of }\left[C_{p} /\left(C_{v}-1\right)\right] /\left(C_{p} / C_{v}\right) \\
\eta= & \text { compressor efficiency } \\
\tau= & \text { pay-out time }(\mathrm{yr}) \\
\mathrm{M} \& \mathrm{~S}= & \text { Marshall and } \mathrm{Swift} \text { index }
\end{aligned}
$$

\section{Positive and Binary Variables}

$$
\begin{aligned}
A C_{g}^{(\mathrm{O})}= & \text { annual operating cost of compressor for } \\
& \text { gas stream } g \\
A C_{g}^{(\mathrm{I})}= & \text { annual investment cost of compressor for } \\
& \text { gas stream } g \\
A C_{g}^{(\mathrm{G})}= & \text { annual cost of pressure vessel } g \\
A C_{l}^{(\mathrm{L})}= & \text { annual cost of liquid vessel } l \\
\mathrm{bhp} & =\text { brake horsepower of gas streams } g \\
D_{g}= & \text { diameter of gas pressure vessel } g(\mathrm{~m}) \\
D_{l}= & \text { diameter of liquid vessel } l(\mathrm{~m}) \\
f p_{g}= & \text { pressure correction factor for pressure } \\
& \text { vessel } g \\
G S_{i p}^{(\mathrm{in})}= & \text { mass flow rate of rich stream } i \text { flows into } \\
& \text { storage } i \text { at period } p(\mathrm{~kg} / \mathrm{h}) \\
\mathrm{hp}_{g p}= & \text { theoretical horsepower of gas streams } \\
& g \text { at period } p \\
L_{a j p}= & \text { mass flow rate of lean stream } j \text { at period } \\
& p \text { in plant } a \text { (kg/h) } \\
H_{j p}^{(\mathrm{in})}= & \text { mass flow rate of lean stream } j \text { flows into } \\
H_{g}= & \text { mass flow rate of storage } i \text { flows into } j \text { at period } p(\mathrm{~kg} / \mathrm{h}) \\
& \text { rich stream } i \text { at period } p(\mathrm{~kg} / \mathrm{h}) \\
& \text { height of liquid vessel } l(\mathrm{~m}) \\
&
\end{aligned}
$$




$$
\begin{aligned}
& L S_{j p}^{(\text {out })}=\text { mass flow rate of storage } j \text { flows into rich } \\
& \text { stream } j \text { at period } p(\mathrm{~kg} / \mathrm{h}) \\
& P_{g}^{(\text {out })}=\text { output pressure of compressor for } \\
& \text { gas stream } g \text { (atm) } \\
& Q_{g, \max }^{(\text {TankG) }}=\text { size of gas pressure vessel } g(\mathrm{~kg}) \\
& Q_{l, \text { max }}^{(\text {TankL) }}=\text { size of liquid vessel } l(\mathrm{~kg}) \\
& Q_{i p}^{(\text {TankR) }}=\text { existent quantity of storage } i \text { at time point } \\
& p(\mathrm{~kg}) \\
& Q_{j p}^{\text {(TankS) }}=\text { existent quantity of storage } j \text { at time point } \\
& p(\mathrm{~kg}) \\
& S_{g p}^{(\mathrm{in})}=\text { input flow rate of compressor } g \text { at time point } \\
& p(\mathrm{~kg}) \\
& z_{g}^{(\text {TankG) }} \in\{0,1\} \text {; the value } 1 \text { denotes the existence of } \\
& \text { storage tank on gas stream } g \\
& z_{l}^{\text {(TankL) }} \in\{0,1\} \text {; the value } 1 \text { denotes the existence of } \\
& \text { storage tank on liquid stream } l \\
& z_{g s} \in\{0,1\} \text {; the value } 1 \text { means that a pressure value } \\
& \text { is located in stage } s \text { of gas stream } g \\
& z r_{i p}^{\text {(in) }} \in\{0,1\} \text {; the value } 1 \text { denotes the existence of rich } \\
& \text { stream } i \text { flowing into storage } i \text { at period } p \\
& z r_{i p}^{\text {(out) }} \in\{0,1\} \text {; the value } 1 \text { denotes the existence of } \\
& \text { storage } i \text { flowing into rich stream } i \text { at period } p \\
& z s_{i p}^{\text {(in) }} \in\{0,1\} \text {; the value } 1 \text { denotes the existence of } \\
& \text { lean stream } j \text { flowing into storage } j \text { at period } p \\
& z s_{i p}^{\text {(out) }} \in\{0,1\} \text {; the value } 1 \text { denotes the existence of } \\
& \text { storage } j \text { flowing into rich stream } j \text { at period } p
\end{aligned}
$$

4.2.1. Storing Policy To Supply Constant Inlet Rates to Networks. Suppose all rich and lean streams are equipped with their individual storage vessels/tanks for adaptive operation during various intervals. Because the upstream rates are perioddependent, part of the input rich or lean stream rates can be stored temporarily during surplus periods and then released from the storage vessels/tanks late during deficit intervals, for supplying continuous operation for MEN. The exact rates for streams $i$ and $j$ that are flowing into the network $\left(G E_{i}\right.$ and $L E_{j}$, respectively) can be calculated as follows:

$$
\begin{array}{ll}
G E_{i}=\sum_{\forall a \in \mathrm{A}} G_{a i p}-G S_{i p}^{(\mathrm{in})}+G S_{i p}^{(\mathrm{out})} & \\
L E_{j}=\sum_{\forall a \in \mathrm{A}} L_{a j p}-L S_{j p}^{(\mathrm{in})}+L S_{j p}^{(\mathrm{out})} & \\
& \forall j \in \mathrm{NCR}, p \in \mathrm{TP} \\
& \forall j \in \mathrm{NCL}, p \in \mathrm{TP}
\end{array}
$$

where $G S_{i p}^{(\mathrm{in})}, G S_{i p}^{\text {(out) }}, L S_{j p}^{(\mathrm{in})}$, and $L S_{j p}^{\text {(out) }}$ respectively denote the rates for inlet up-rich stream $i$ or up-lean stream $j$ flowing into or out of their storage vessels/tanks during period $p$. For all up or external streams, the exact rates in network are the sum of the input rates of all plants. In addition, to consider continuous operation, the input rates during all periods are equal.

$$
\begin{array}{r}
G E_{i}=\sum_{\forall a \in \mathrm{A}} G_{a i p}, G_{a i p}=G_{a i p^{\prime}} \\
\forall a \in \mathrm{A}, i \in \mathrm{CR}, p, p^{\prime} \in \mathrm{TP} \\
L E_{j}=\sum_{\forall a \in \mathrm{A}} L_{a j p}, L_{a j p}=L_{a j p^{\prime}} \\
\forall a \in \mathrm{A}, j \in \mathrm{CL}, p, p^{\prime} \in \mathrm{TP}
\end{array}
$$

4.2.2. Remaining Mass for Transferable Components for Each Storage. The remaining mass of each storage vessel/ tank at the end of each period $p$ is relevant for designing a suitable tank size. The remaining mass of each storage $i$ at the end of period $p\left(Q_{i, p+1}^{(\mathrm{TankR})}\right)$ is equal to the tank's initial mass $\left(Q_{i, p}^{(\text {TankR })}\right)$ plus the input mass from its corresponding stream minus the output mass from the storage vessel/tank. Note also that the remaining mass of each storage $i$ at period $p$ is also the initial mass of the storage at subsequent period $p$ +1 . These constraints also apply for storage tanks for lean streams.

$$
\begin{gathered}
Q_{i, p+1}^{(\text {TankR) }}=Q_{i p}^{(\text {TankR })}+\left(G S_{i p}^{(\text {in })}-G S_{i p}^{(\text {out })}\right) t_{p} \\
\forall i \in \mathrm{NCR}, p \in \mathrm{TP} \\
\begin{array}{c}
Q_{j, p+1}^{(\text {TankS })}=Q_{j p}^{(\text {TankS })}+\left(L S_{j p}^{(\text {in })}-L S_{j p}^{(\text {out })}\right) t_{p} \\
\forall j \in \mathrm{NCL}, p \in \mathrm{TP}
\end{array}
\end{gathered}
$$

Here, $t_{p}$ is the elapsed time of period $p$. Also note that both of the initial mass at the first time point $(p=1)$ and the remaining mass at the final time point $(p+1)$ should be equal for cyclic operations. Thus, the initial mass at the first time point $(p=1)$ can be stated as follows:

$$
\begin{array}{ll}
Q_{i 1}^{(\text {TankR) }}=Q_{i, N_{p}}^{\text {(Tank) }}+\left(G S_{i, N_{p}}^{\text {(in) }}-G S_{i, N_{p}}^{(\text {out })}\right) t_{N_{p}} & \\
& \forall i \in \text { NCR (26a) } \\
Q_{j 1}^{\text {(TankS) }}=Q_{j, N_{p}}^{\text {(TankS) }}+\left(L S_{j, N_{p}}^{\text {(in) }}-L S_{j, N_{p}}^{\text {(out) }}\right) t_{N_{p}} & \\
& \forall j \in \text { NCL (26b) }
\end{array}
$$

The binary variables $z r_{i p}^{\text {(in) }}$ and $z r_{i p}^{\text {(out) }}$ (or $z s_{j p}^{\text {(in) }}$ and $z s_{j p}^{\text {(out) }}$ ) are used to denote the status of rich stream $i$ (or lean stream $j$ ) flowing into (or releasing from) its own storage vessel/tank. The stored rate has the normal input rate as its upper bound; and the release rate is also limited by the remainder in the storage vessel/tank. Meanwhile, the actions of storage and discharge are disjunctive at the same time. The following equations summarize these restraints:

$$
\left.\begin{array}{l}
G S_{i p}^{\text {(in) }}-\sum_{\forall a \in \mathrm{A}} G_{a i p} z r_{i p}^{(\text {in })} \leq 0 \\
G S_{i p}^{\text {(out) }} t_{p}-Q_{i p}^{\text {(TankR) }} z r_{i p}^{\text {(out) }} \leq 0 \\
z r_{i p}^{\text {(in) }}+z r_{i p}^{\text {(out) }} \leq 1
\end{array}\right\} \quad \forall i \in \mathrm{NCR}, p \in \mathrm{TP}
$$

4.2.3. Bounds on Variables. Lean streams may possibly come from the process; bounds for available mass of lean process streams then should be set.

$$
L_{a j p} \leq L_{a j p}^{(\mathrm{up})} \quad \forall a \in \mathrm{A}, j \in \mathrm{LP}, p \in \mathrm{TP}
$$




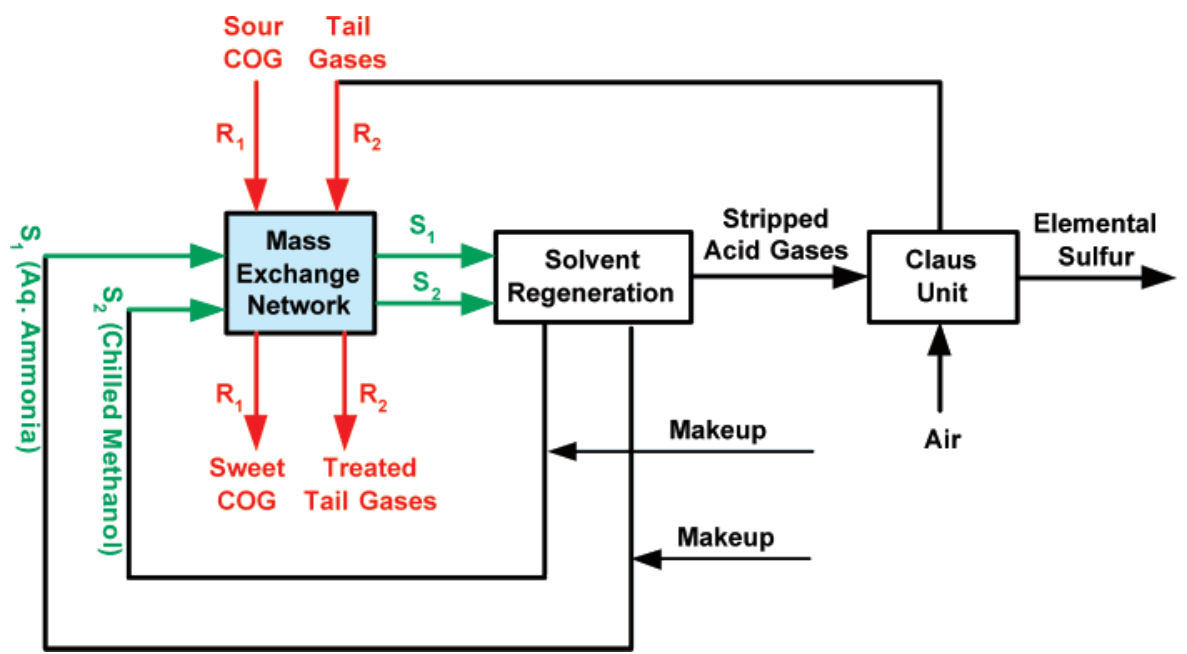

Figure 3. Schematic of the coke oven gas (COG) sweetening batch process.

Table 1. Stream Data for the Semiconsecutive Coke Oven Gas (COG) Process

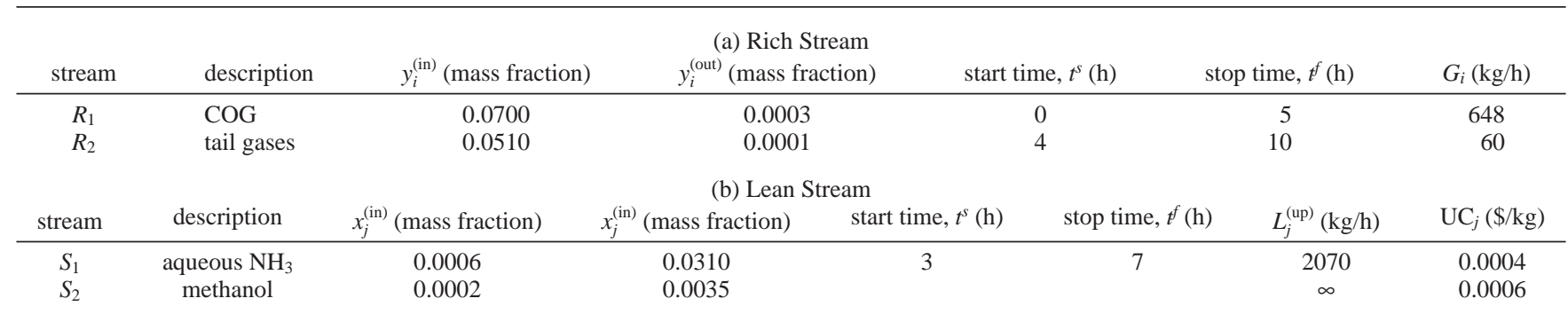

cost of plate column $=4552 N \$ / \mathrm{yr}\left(D=1 \mathrm{~m}\right.$, for $S_{1}$ and $\left.S_{2}\right)$

Table 2. Physical Properties of Gas and Liquid Streams

\begin{tabular}{|c|c|c|c|c|}
\hline \multicolumn{5}{|c|}{ (a) Gas Streams } \\
\hline stream & $\begin{array}{c}\text { gas pressure, } \\
P_{\mathrm{g}}^{\text {(in) }} \\
\end{array}$ & $\begin{array}{c}\text { density of gas, } \\
\rho_{g}^{\text {(in) }} \\
\end{array}$ & $F \mathrm{~m}_{\mathrm{g}}$ & $F \mathrm{c}_{g}$ \\
\hline$R_{1}$ & 2 & 0.4 & 3.67 & 1.82 \\
\hline$R_{2}$ & 2 & 1.2 & 3.67 & 1.82 \\
\hline \multicolumn{5}{|c|}{ (b) Liquid Streams } \\
\hline stream & $\begin{array}{l}\text { liquid pressure, } \\
\qquad P_{l}^{\text {(in) }}\end{array}$ & \multicolumn{2}{|c|}{$\begin{array}{l}\text { density of liquid, } \\
\rho_{l}^{\text {(in) }}\end{array}$} & $\mathrm{F} m_{l}$ \\
\hline$S_{1}$ & 1 & \multicolumn{2}{|c|}{892} & 3.67 \\
\hline$S_{2}$ & 1 & \multicolumn{2}{|c|}{792} & 3.67 \\
\hline
\end{tabular}

Table 3. Pressure Factors

\begin{tabular}{lllccccccccc}
\hline$s$ & $P_{s}$ & $F_{s}$ & $s$ & $P_{s}$ & $F_{s}$ & $s$ & $P_{s}$ & $F_{s}$ & $s$ & $P_{s}$ & $F_{s}$ \\
\hline 1 & 0 & 0 & 4 & 13.6 & 1.15 & 7 & 34.0 & 1.45 & 10 & 54.4 & 1.9 \\
2 & 3.4 & 1 & 5 & 20.4 & 1.2 & 8 & 40.8 & 1.6 & 11 & 61.2 & 2.3 \\
3 & 6.8 & 1.05 & 6 & 27.2 & 1.35 & 9 & 47.6 & 1.8 & 12 & 68.0 & 2.5
\end{tabular}

4.2.4. Gas Compressor. The design equation for the theoretical horsepower (hp) for a gas compressor $g$ added on gas stream $g$ is shown in eq $29:{ }^{20}$

$$
\begin{aligned}
\mathrm{hp}_{g p}=\left(\frac{0.038}{\gamma}\right) P_{g}^{(\mathrm{in})}\left(\frac{S_{g p}^{(\mathrm{in})}}{\rho_{g}^{(\mathrm{in})}}\right) & {\left[\left(\frac{P_{g}^{(\text {out })}}{P_{g}^{(\mathrm{in})}}\right)^{\gamma}-1\right] } \\
\forall g & \in \mathrm{NCR}^{(\mathrm{gas})} \cup \mathrm{NCL}^{(\mathrm{gas})}, p \in \mathrm{TP}
\end{aligned}
$$

where $S_{g p}^{(\mathrm{in})}$ is the input flow rate of compressor $g$ at period $p$. When the stream is a gas-phase rich stream, $S_{g p}^{(\mathrm{in})}=G E_{i p}^{(\mathrm{in})}$; when the stream is a gas-phase lean stream, $S_{g p}^{(\mathrm{in})}=L E_{j p}^{(\mathrm{in})}$.

$$
S_{g p}^{(\mathrm{in})}= \begin{cases}G S_{i p}^{(\mathrm{in})} & i, g \in \mathrm{NCR}^{(\mathrm{gas})}, p \in \mathrm{ETP} \\ L S_{j p}^{(\mathrm{in})} & j, g \in \mathrm{NCL}^{(\mathrm{gas})}, p \in \mathrm{TP}\end{cases}
$$

We should limit the upper and lower bounds for the output pressure of compressor $g$, when the pressure vessel $g$ is existing (i.e., $z_{g}^{(\text {TankG) }}=1$ ). Moreover, the output pressure should be equal to the input pressure, because the gas vessel $g$ is nonexistent (i.e., $z_{g}^{(\text {TankG) }}=0$ ).

$$
\begin{aligned}
z_{g}^{(\text {TankG) }} & P_{g}+\left(1-z_{g}^{(\text {TankG) })} P_{g}^{(\text {in })} \leq P_{g}^{\text {(out) }} \leq z_{g}^{(\text {TankG })} \bar{P}_{g}+\right. \\
& \left(1-z_{g}^{(\text {TankG })}\right) P_{g}^{(\text {in })} \quad \forall g \in \mathrm{NCR}^{(\text {gas })} \cup \mathrm{NCL}^{\text {(gas) }}
\end{aligned}
$$

By dividing the brake horsepower by the driver efficiency and timing of the elapsed time at each period, we can calculate the electricity requirement during period $p$. Then, by summing them for periods and multiplying by the electric cost and the batch number, we can determine the annual operating cost of compressor $g$.

$$
\begin{aligned}
& A C_{g}^{(\mathrm{O})}=N_{B} \mathrm{Chp} \sum_{\forall p \in \mathrm{TP}} \frac{\mathrm{hp}_{g p} t_{p}}{\eta} \\
& \forall g \in \mathrm{NCR}^{(\text {gas })} \cup \mathrm{NCL}^{\text {(gas) }}
\end{aligned}
$$

The maximum brake horsepower of gas stream $g$ during entire periods $\left(\mathrm{bhp}_{g}\right)$ is used to calculate the installed cost of compressor $g$ : 


$$
\begin{array}{ll}
\mathrm{bhp}_{g} \geq \frac{\mathrm{hp}_{g p}}{\eta} & \forall g \in \mathrm{NCR}^{\text {(gas) }} \cup \mathrm{NCL}^{(\text {gas })}, p \in \mathrm{TP} \\
A C_{g}^{(\mathrm{I})}=\frac{(\mathrm{M} \& \mathrm{~S} / 280)(517.5)\left(\mathrm{bhp}_{g}\right)^{0.82}\left(2.11-F \mathrm{c}_{g}\right)}{\tau} & \forall g \in \mathrm{NCR}^{\text {(gas) }} \cup \mathrm{NCL}^{\text {(gas) }}
\end{array}
$$

where $F \mathrm{c}_{g}$ is the correction factor for compressor of gas stream $g$ and M\&S is the Marshall and Swift inflation index. To determine the installed cost on an annual basis, we introduce a capital charge factor, $\tau$.

4.2.5. Pressure Vessel. If the pressure vessel $g$ exists (i.e., $\left.z_{g}^{(\text {TankG) }}=1\right)$, we need variable $Q_{g, \max }^{(\text {TankG) }}$ to design the size, according to its density and pressure compression. Note that we assume the shape of vessel is circular.

$$
\begin{aligned}
& Q_{g, \max }^{(\text {TankG) }} \geq Q_{g p}^{(\text {TankG) }} \quad \forall g \in \mathrm{NCR}^{\text {(gas) }} \cup \mathrm{NCL}^{\text {(gas) }}, p \in \mathrm{TP} \\
& \underline{U} z_{g}^{(\text {TankG) }} \leq Q_{g p}^{(\text {TankG) }} \leq \bar{U} z_{g}^{(\text {TankG) }} \\
& \forall g \in \mathrm{NCR}^{\text {(gas) }} \cup \mathrm{NCL}^{\text {(gas) }}, p \in \mathrm{TP} \\
& \left(\frac{Q_{g, \max }^{\text {(Tank) }}}{\rho_{g}^{(\text {in })}}\right)\left(\frac{P_{g}^{(\text {in })}}{P_{g}^{(\text {out })}}\right)=\frac{\pi}{4} D_{g}^{2} H_{g} \\
& \forall g \in \mathrm{NCR}^{\text {(gas) }} \cup \mathrm{NCL}^{\text {(gas) }}
\end{aligned}
$$

Here, $Q_{g p}^{(\text {TankG) }}$ is the quantity of pressure vessel $g$ at period $p$. When the stream is a gas-phase rich stream, $Q_{g p}^{(\mathrm{TankG})}=$

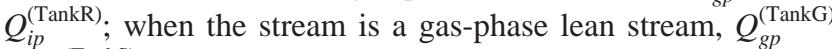
$=Q_{j p}^{(\mathrm{Tank})}$

$$
Q_{g p}^{(\text {TankS })}= \begin{cases}Q_{i p}^{(\text {TankR })} & i, g \in \mathrm{NCR}^{(\mathrm{gas})}, p \in \mathrm{TP} \\ Q_{j p}^{(\text {TankS })} & j, g \in \mathrm{NCL}^{(\mathrm{gas})}, p \in \mathrm{TP}\end{cases}
$$

The outlet pressure of compressor $g$ is the pressure of vessel $g$, so the variable will affect the vessel's size by the pressure factor, $f p_{g}$. The binary variable $z_{g s}$ can define the location of output pressure. For example, $z_{g 5}=1$ means the output pressure that is located between $P_{4}$ and $P_{5}$. The output pressure is only located on one stage. Thus, the summation of $z_{g s}$ for stage is 1 , because $f p_{g}$ becomes equal to $F_{s}$ as $z_{g s}=1$.

$$
\begin{gathered}
f p_{g}=\sum_{s \in \mathrm{PS}} F_{s} z_{g s} \quad \forall g \in \mathrm{NCR}^{(\text {gas })} \cup \mathrm{NCL}^{\text {(gas })} \\
\sum_{s \in \mathrm{PS}} z_{g s}=1 \quad \forall g \in \mathrm{NCR}^{\text {(gas) }} \cup \mathrm{NCL}^{\text {(gas) }} \\
P_{s-1}-\bar{P}_{g}\left(1-z_{g s}\right) \leq P_{g}^{\text {(out) }} \leq P_{s}+\bar{P}_{g}\left(1-z_{g s}\right) \\
\forall g \in \mathrm{NCR}^{\text {(gas) }} \cup \mathrm{NCL}^{\text {(gas) }}, s \in \mathrm{PS}
\end{gathered}
$$

Thus, the cost of pressure vessel $g$ can be calculated:

$$
\begin{array}{r}
A C_{g}^{(\mathrm{G})}=\frac{(\mathrm{M} \& \mathrm{~S} / 280)\left(957.8820 D_{g}{ }^{1.066} H_{g}^{0.82}\left(2.18+F \mathrm{~m}_{g} f p_{g}\right)\right)}{\tau} \\
\forall g \in \mathrm{NCR}^{\text {(gas) }} \cup \mathrm{NCL}^{\text {(gas) }} \text { (35) }
\end{array}
$$

where $F \mathrm{~m}_{g}$ is the material correction factor of pressure vessel $g$.

4.2.6. Liquid Tank. For liquid tanks, the input pressure is almost equal to its output pressure. Therefore, it does not need a compressor, and its size is not related to the output pressure.

$$
\begin{aligned}
& Q_{l, \max }^{(\text {Tank })} \geq Q_{l p}^{(\text {TankL) }} \quad \forall l \in \mathrm{NCR}^{\text {(liq) }} \cup \mathrm{NCL}^{\text {(liq) }}, p \in \mathrm{TP} \\
& \underline{U} z_{l}^{(\text {TankL) }} \leq Q_{l p}^{(\text {TankL) }} \leq \bar{U} z_{l}^{(\text {TankL) }} \\
& \forall l \in \mathrm{NCR}^{\text {(liq) }} \cup \mathrm{NCL}^{\text {(liq) }}, p \in \mathrm{TP} \\
& \frac{Q_{l, \max }^{(\text {TankL) }}}{\rho_{l}^{\text {(in) }}}=\frac{\pi}{4} D_{l}^{2} H_{l} \quad \forall l \in \mathrm{NCR}^{\text {(liq) }} \cup \mathrm{NCL}^{\text {(liq) }}
\end{aligned}
$$

Here, $Q_{l p}^{\text {(TankL) }}$ is the quantity of tank $l$ at period $p$. When the stream is one liquid-phase rich stream, $Q_{l p}^{\text {(TankL) }}=Q_{i p}^{\text {(TankR); }}$ when the stream is one liquid-phase lean stream, $Q_{l p}^{(\text {TankL) }}=$ $Q_{j p}^{\text {(TankS) }}$.

$$
Q_{l p}^{(\text {TankL })}= \begin{cases}Q_{i p}^{(\text {TankR })} & i, l \in \mathrm{NCR}^{(\mathrm{liq})}, p \in \mathrm{TP} \\ Q_{j p}^{(\text {TankS })} & j, l \in \mathrm{NCL}^{(\mathrm{liq})}, p \in \mathrm{TP}\end{cases}
$$

The main difference of cost between the gas vessel and the liquid tank is the pressure factor. The pressure factor is always 1 for liquid tanks. The cost function of liquid tanks can be given as follows:

$$
\begin{array}{r}
A C_{l}^{(\mathrm{L})}=\frac{(\mathrm{M} \& \mathrm{~S} / 280)\left(957.8820 D_{l}^{1.066} H_{l}^{0.82}\left(2.18+F \mathrm{~m}_{l}\right)\right)}{\tau} \\
\forall l \in \mathrm{NCR}^{\text {(liq) }} \cup \mathrm{NCL}^{\text {(liq) }}
\end{array}
$$

4.3. Sequential Design Objectives and MINLP Formulations. In the sequential design approach, the network configuration and the operational rates of all streams will be resolved initially (Phase I) by minimizing the TAC around the continuous MEN. Therein, the mass rates of lean/rich streams will also be determined and then passed to the second design phase. The second design phase follows to minimize the cost of streams storage, based on the network configuration and the streams flow rates supplied by the first design phase.

4.3.1. Design Phase I. The TAC in design phase $\mathrm{I}\left(\mathrm{TAC}_{N}\right)$ involves a combination of operating costs of MSAs and the tray and/or height costs for each exchanger.

$$
\begin{gathered}
\min _{x_{N} \in \Omega_{N}} \mathrm{TAC}_{N}=N_{\mathrm{B}} \sum_{\forall j \in \mathrm{LP}} \sum_{\forall p \in \mathrm{TP}} \mathrm{UC}_{j} L E_{j} t_{p}+ \\
\sum_{\forall i \in \mathrm{RP}} \sum_{\forall j \in \mathrm{LP}^{(\mathrm{rr})} \sum_{\forall k \in \mathrm{ST}} A C_{i j}^{(\mathrm{tr})} N_{i j k}+} \sum_{\forall i \in \mathrm{RP}} \sum_{\forall j \in \mathrm{LP}} \sum_{\forall k \in \mathrm{ST}} A C_{i j}^{(\mathrm{h})} H_{i j k} \\
\left(\text { for } \Omega_{N}=\left\{x_{N} \mid \text { set of constraints (eqs 1-22) }\right\}\right)
\end{gathered}
$$

where $x_{N}$ and $\Omega_{N}$ denote the design variables and the feasible space relevant to the continuous MEN (eqs 1-22), respectively.

4.3.2. Design Phase II. The subsequent design objective in the second phase is to minimize the cost of streams storage, based on the network configuration resolved in Design Phase I. The cost concerned in Design Phase II $\left(\mathrm{TAC}_{S}\right)$ consists of 


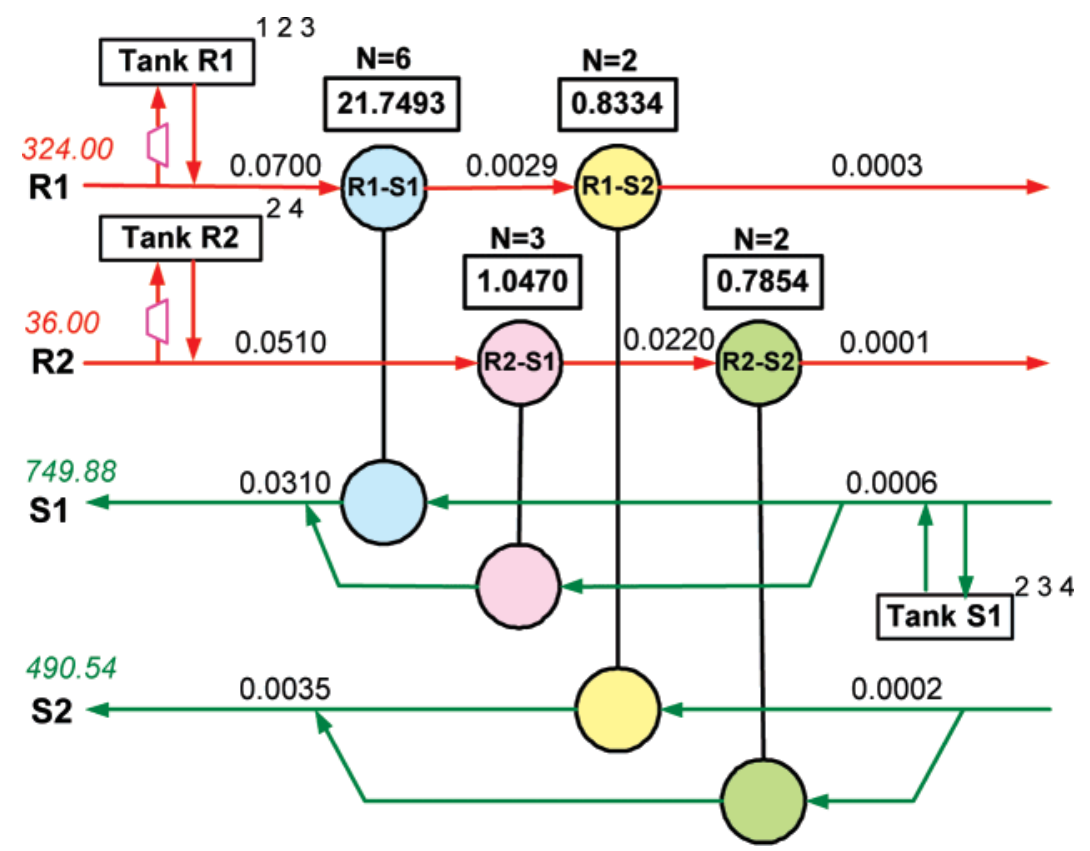

Figure 4. Schematic of the resulting MEN configuration with storage pressure vessels and a liquid tank. The superscripts beside the tanks indicate the case studies for which the tanks are required.

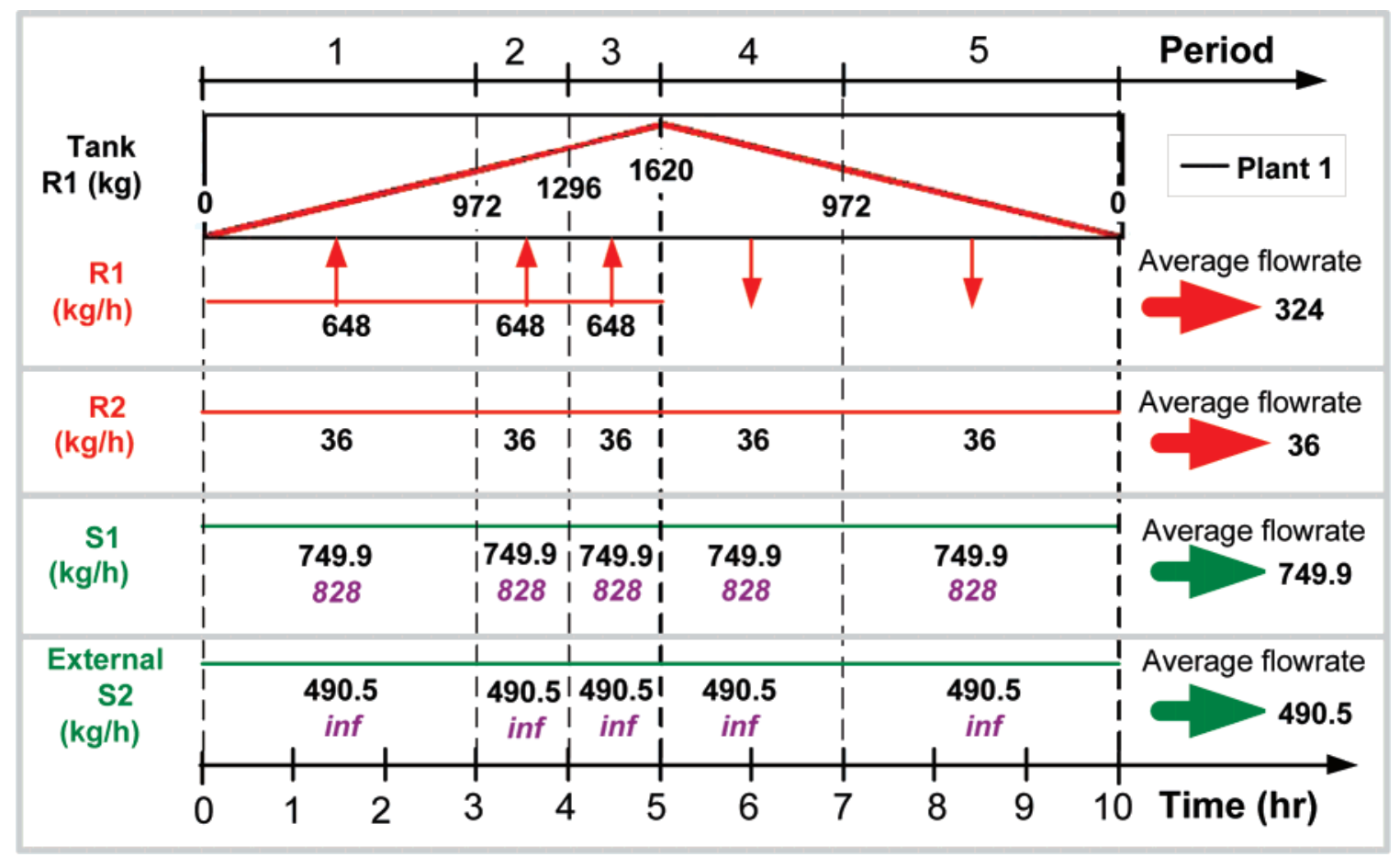

Figure 5. Remainder in pressure vessel $R_{1}$ and the storing policy for case 1 .

the operating cost and the annualized investment cost of compressors, pressure vessels for gas streams, and liquid tanks.

$$
\begin{gathered}
\min _{x_{S} \in \Omega_{S}} \mathrm{TAC}_{S}=\sum_{\substack{\forall g \in \mathrm{NCR}^{\text {(gas) }} \cup \mathrm{NCL}^{\text {(gas) }}\\
}}\left(A C_{g}^{(\mathrm{O})}+A C_{g}^{(\mathrm{I})}+A C_{g}^{(\mathrm{G})}\right)+ \\
\sum_{\substack{\forall l \in \mathrm{NCR}^{(\text {liq })} \\
\cup N C L^{\text {(liq) }}}} A C_{l}^{(\mathrm{L})}
\end{gathered}
$$$$
\text { (for } \Omega_{S}=\left\{x_{S} \mid \text { set of constraints (eqs 23-37) }\right\} \text { ) }
$$

Notably, the batch MEN synthesis problem also can be resolved by simultaneous optimization, where the total annual cost of the overall system (TAC) is used as the design objective, $x$ represents the design variables $\left(x=x_{N} \cup x_{S}\right)$, and and $\Omega$ represents the feasible searching space $\left(\Omega=\Omega_{N} \cap \Omega_{S}\right)$.

$$
\min _{x \in \Omega} \mathrm{TAC}=\mathrm{TAC}_{N}+\mathrm{TAC}_{S}
$$

The solution of eq 40 might be time-consuming. Although direct solution of eq 40 can provide the optimal design of the overall system, the sequential design approach is applied to simplify the solution procedure. The supplied numerical example will demonstrate that the sequential design approach can usually provide a satisfactory solution. 


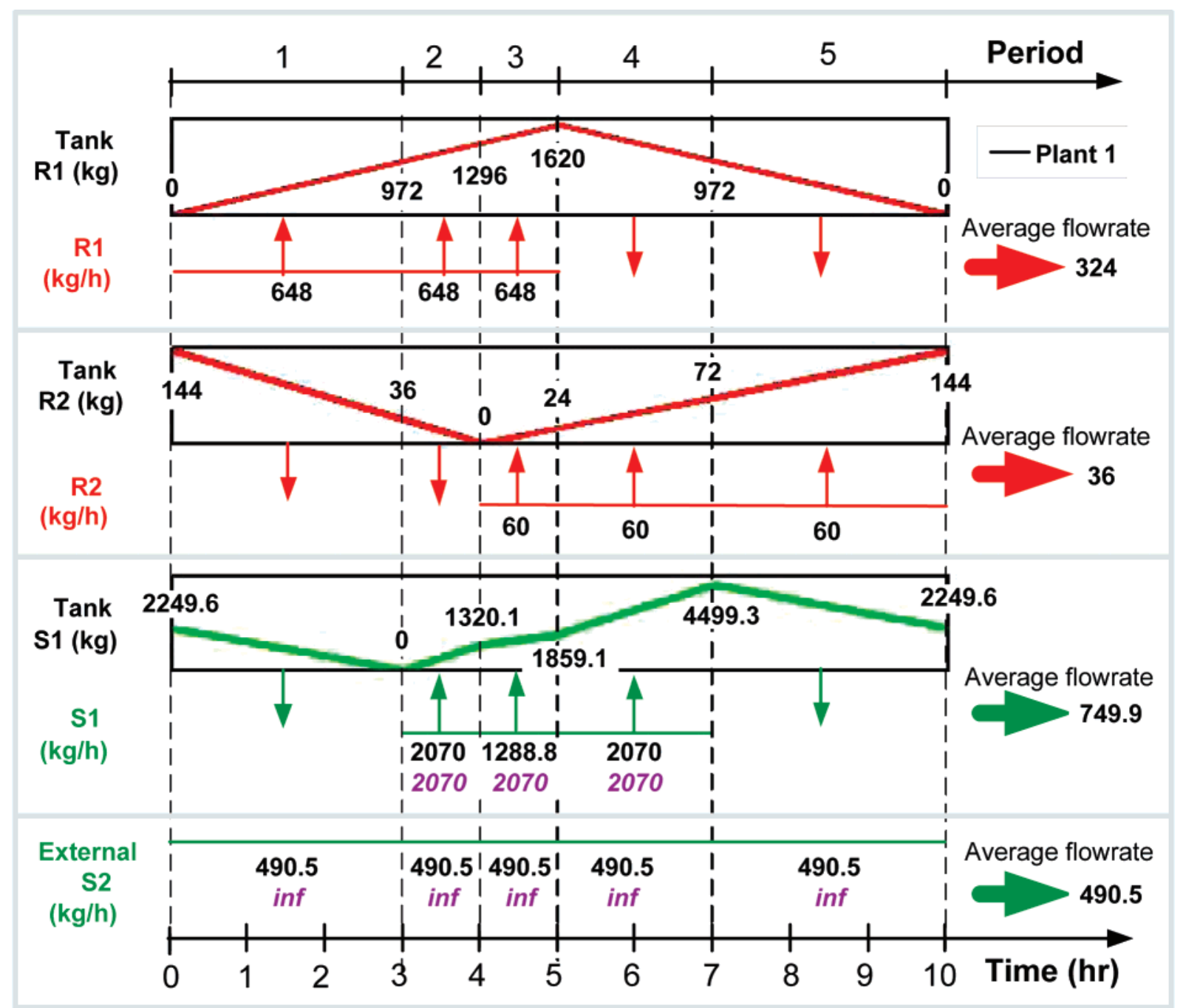

Figure 6. Remainders in pressure vessels $R_{1}$ and $R_{2}$ and liquid tank $S_{1}$, and their storing policy for case 2 .

Table 4. Detailed Costs and Sizes of Compressors and Pressure Vessels

\begin{tabular}{|c|c|c|c|c|}
\hline parameter & case 1 & case 2 & case 3 & case 4 \\
\hline Network Cost (\$/yr) & 107610 & 107610 & 107610 & 107610 \\
\hline MSA (\$/yr) & 48434 & 48434 & 48434 & 48434 \\
\hline exchangers $(\$ / y r)$ & 59176 & 59176 & 59176 & 59176 \\
\hline Storing Cost $(\$ / y r)$ & 473628 & 529739 & 163111 & 24065 \\
\hline compressor R1 & 211432 & 211432 & 58704 & \\
\hline operating (\$/yr) & 33400 & 33400 & 11133 & \\
\hline investment (\$/yr) & 178032 & 178032 & 47571 & \\
\hline pressure (atm) & 34 & 34 & 34 & \\
\hline compressor R2 & & 10181 & & 8858 \\
\hline operating (\$/yr) & & 1068 & & 330 \\
\hline investment (\$/yr) & & 9113 & & 8558 \\
\hline pressure (atm) & & 40 & & 34 \\
\hline pressure vessel R1 & 262196 & 262196 & 95325 & \\
\hline diameter (m) & 5.33 & 5.33 & 3.11 & \\
\hline maining $(\mathrm{kg})$ & 1620 & 1620 & 324 & \\
\hline pressure vessel R2 & & 27812 & & 9304 \\
\hline diameter $(m)$ & & 1.56 & & 0.91 \\
\hline maximum rema & & 144 & & 24 \\
\hline liquid tank (S1) & & 18118 & 9081 & 5874 \\
\hline diameter $(\mathrm{m})$ & & 1.48 & 1.02 & 0.81 \\
\hline maximum remaining & & 4499 & 1500 & 750 \\
\hline Total Annual Cost, TAC & 581238 & 637349 & 270721 & 131675 \\
\hline
\end{tabular}

\section{Numerical Example: Sweetening of COG}

This example, originated from El-Halwagi and Manousiouthakis, ${ }^{1}$ as depicted in Figure 3, involves the simultaneous removal of $\mathrm{H}_{2} \mathrm{~S}(n=1)$ and $\mathrm{CO}_{2}(n=2)$ from the coke oven gas (COG) (where $R_{1}$ is a mixture of $\mathrm{H}_{2}, \mathrm{CH}_{4}, \mathrm{CO}, \mathrm{N}_{2}, \mathrm{NH}_{3}$,
$\mathrm{CO}_{2}$, and $\left.\mathrm{H}_{2} \mathrm{~S}\right)$ and the Claus unit tail gas $\left(R_{2}\right)$. The $\mathrm{H}_{2} \mathrm{~S}$ should be removed when the COG is used as a fuel to reduce the emission of corrosive $\mathrm{SO}_{2}$. The presence of ammonia in COG lead to the utilization of aqueous ammonia as a process lean stream $\left(S_{1}\right)$. The chilled methanol is used as an external MSA $\left(S_{2}\right)$. The equilibrium solubility data for $\mathrm{H}_{2} \mathrm{~S}$ in aqueous ammonia and methanol can be correlated using the following relations:

$y_{i}= \begin{cases}1.45 x_{1} & \text { in aqueous ammonia } \\ 0.26 x_{2} & \text { in methanol }\end{cases}$

(where $i=1$ (COG), 2 (tail gas))

Perforated-plate columns are used for both solvents, and a minimum composition difference of $\epsilon=0.0001$ is selected to determine a feasible mass exchange at the inlet and outlet of each potential mass-exchange unit. The basic features of the process and problem data are provided in Table $1,{ }^{10}$ including input and output mass fractions, starting and ending times, mass flow rates during the existing time period for streams, and unit cost, which is modified from the data for continuous operational scenario. ${ }^{1}$ Notably, the regeneration cost of aqueous ammonia has been considered here. Thus, the aqueous ammonia is not free, although it comes from the COG process itself. Also note that the units of MSA cost referenced by El-Halwagi and Manousiouthakis ${ }^{1}$ ( $\$ \mathrm{~s} / \mathrm{kg} \mathrm{yr}$ ) are multiplied by the term $\mathrm{yr} /(8150$ $\times 3600)$ s to convert to units of $\$ / \mathrm{kg}$. 

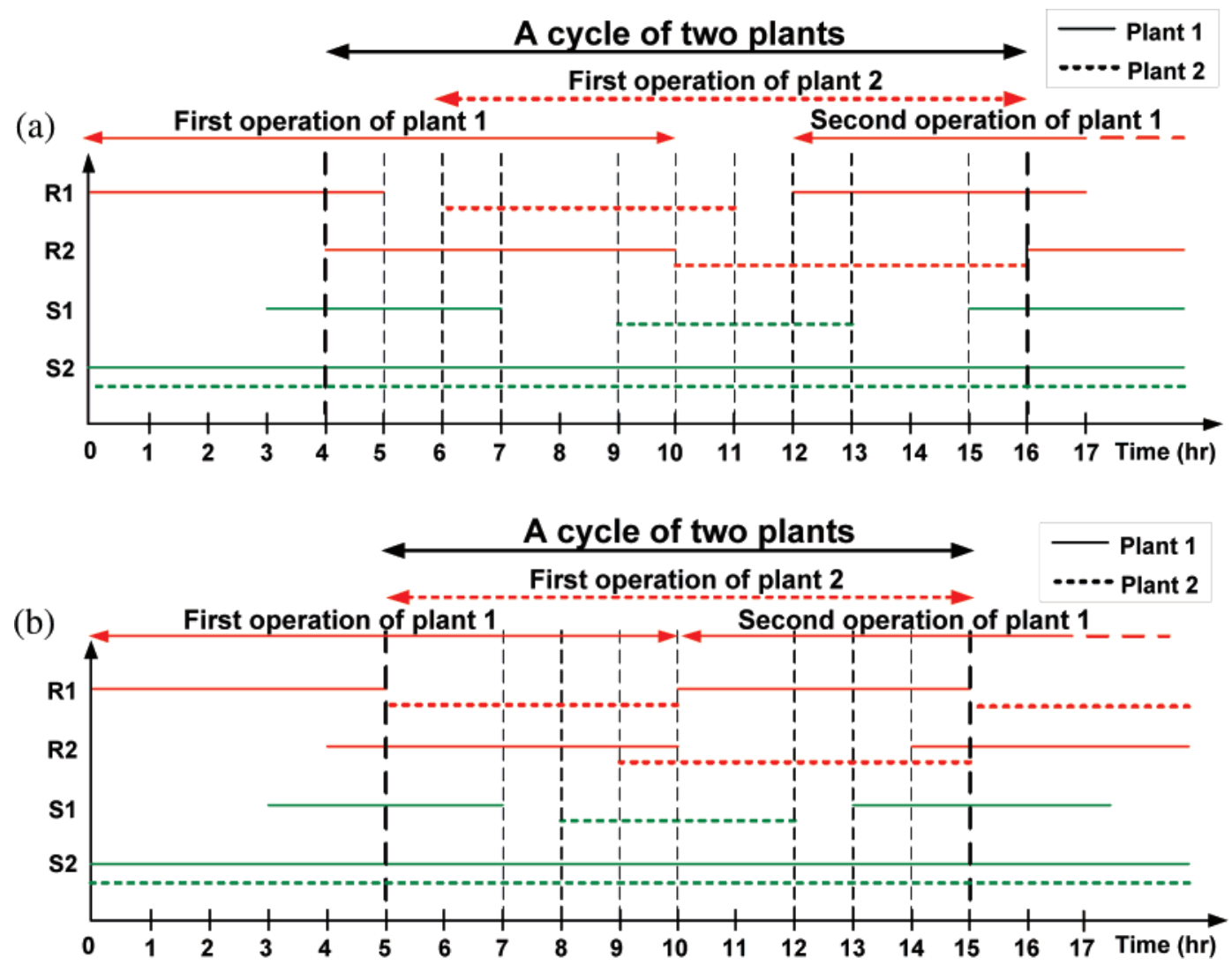

Figure 7. Two parallel COG process plants with (a) longer cyclic times (12 h) and (b) shorter cyclic times (10 h).

The physical properties of gas and liquid streams are shown in Table 2, and the pressure factor is given in Table 3. Some important parameters are set as following: $M \& S=1231.4, \gamma$ $=0.23$ (for more-complex gases), $\eta=0.9, \tau=5 \mathrm{yr}$, Chp $=$ $0.03(\$ /(\mathrm{hp} \mathrm{h}))$, and $N_{\mathrm{B}}=8150(\mathrm{~h} / \mathrm{yr}) /($ one cycle time $(\mathrm{h}))$. In addition, we assume that the height of each vessel/tank is twice its diameter.

To solve the MINLP formulation for the continuous MEN synthesis model following the semiconsecutive COG process, the General Algebraic Modeling System (GAMS) ${ }^{19}$ is used as the main solution tool. The MINLP and nonlinear programming (NLP) solvers are BARON and SNOPT, respectively. Four different operating scenarios are investigated in the following discussion, including the case for the original semiconsecutive COG process (case 1), the modified COG process with three semiconsecutive streams (case 2), and two parallel COG plants with longer cycle times (case 3 ) and shorter cycle times (case 4).

5.1. Case 1: Continuous MEN Synthesis for the Original Semiconsecutive COG Process. In the original semiconsecutive COG process, there are three noncontinuous streams (the COG $\left(R_{1}\right)$, the Claus unit tail gas $\left(R_{2}\right)$, and the process lean stream $\left(S_{1}\right)$ ), and one continuous stream $S_{2}$, which is the external MSA. However, $R_{2}$ and $S_{1}$ are regenerated after going through the MEN. Hence, when the MEN is operated continuously in cyclic mode, $R_{2}$ and $S_{1}$ are provided continuously from the regeneration process and then turn out to be continuous streams. Therefore, there is only one noncontinuous stream $\left(R_{1}\right)$ that requires a storage tank for continuous operation.

Figure 4 shows the final network configuration, which consists of four mass-exchange units, one pressure vessel, and an associated compressor for $R_{1}$. Notably, investigation of the latter cases will show that the final network configuration is the same for all four cases. Furthermore, a pressure vessel and compressor for $R_{1}$ is needed for cases $1-3$, that for $R_{2}$ is required for cases 2 and 3 , and tank $S_{1}$ is used for cases $2-4$, as indicated in this figure. The mass loads and tray numbers, stream flow rates, and compositions are also marked in the figure. Figure 5 shows the operating policy, where the average flow rate for $R_{1}$ is adapted as $324 \mathrm{~kg} / \mathrm{h}$. Thus half of the inlet $R_{1}$ during periods $1-3(0-5 \mathrm{~h})$ is compressed and stored in the pressure vessel, and the stored $R_{1}$ is released into the mass-exchange units with a rate of $324 \mathrm{~kg} / \mathrm{h}$ during periods 4 and $5(5-10 \mathrm{~h})$. The maximum amount that remains in vessel $R_{1}$ is $1620 \mathrm{~kg}$. Note that the numerical values below lines $S_{1}$ and $S_{2}$ denote their flow rates and upper limits. Notice that the compression work and the vessel size for $R_{1}$ are dependent on the maximum allowable vessel pressure. Suppose the upper vessel pressure is limited to $40 \mathrm{~atm}$. Table 4 shows the details regarding the costs and vessel sizes. Note that the resulting maximum vessel pressure of tank $R_{1}$ is $34 \mathrm{~atm}$, which is quite high; thus, the pressure vessel cost comprises almost half of the TAC.

5.2. Case 2: Modified COG Process with Three Semiconsecutive Streams. There is only one continuous stream in the COG process that is accompanied by a continuous MEN, as discussed in case 1 . To extend our investigation on continuous MEN synthesis with multiple semiconsecutive inlet streams, we assume that $R_{1}, R_{2}$, and $S_{1}$ remain intermittent in case 2 . To concentrate the discussion on the differences of storing policies, the average flow rates and input/output compositions of streams in case 2 are the same as in the previous case. The MEN configuration of Figure 4 can be used under such conditions. However, the storing policies are quite different for case 2, as shown in Figure 6. In Figure 6, there are two pressure vessels for $R_{1}$ and $R_{2}$ and one liquid tank for $S_{1}$. The storing policy of $R_{1}$ is the same as that of case 1 , because of the same given of 


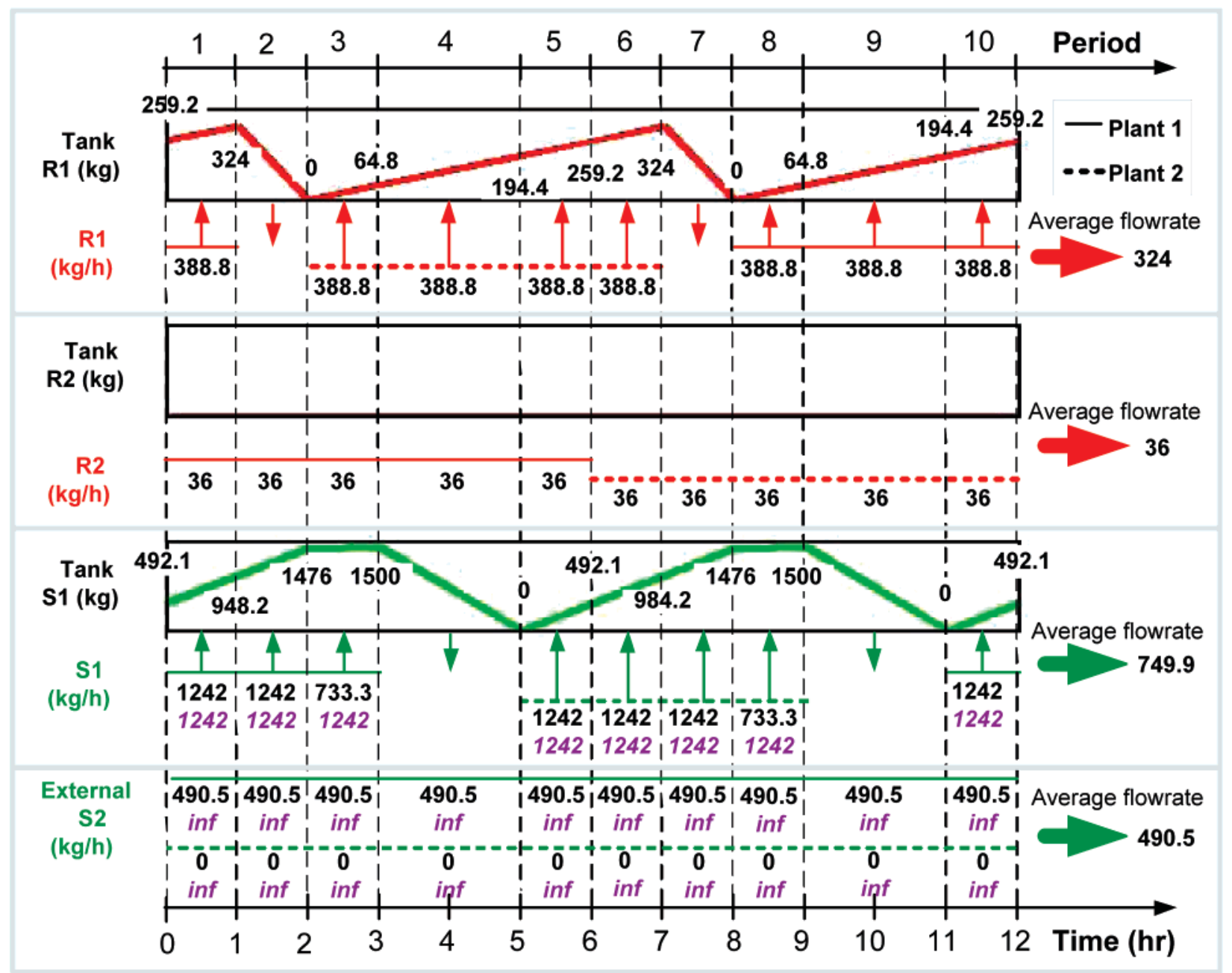

Figure 8. Remainders in vessel $R_{1}$ and liquid tank $S_{1}$ and the storing policies for case 3 .

$R_{1}$. Stream $R_{2}$ flows into the pressure vessel during periods $3-5$ and then discharges into the MEN at periods 1 and 2 of the next cycle. $S_{1}$ flows into tank $S_{1}$ during periods $2-4$. It then is discharged into the MEN during periods 5 and 1 of the next cycle. These policies cause the average flow rates of $R_{2}$ and $S_{1}$ to become $36 \mathrm{~kg} / \mathrm{h}$ and $749.9 \mathrm{~kg} / \mathrm{h}$, respectively. Details of various cost items are also shown in Table 4.

5.3. Case 3: Two Parallel COG Plants with Longer Cyclic Time. We assume two modified COG plants with half the capacity of case 2 operated in turn to supply semiconsecutive inlet streams in sequence. Therefore, the mass flow rates of rich streams and the upper mass flow rates of lean streams are 324 $\mathrm{kg} / \mathrm{h}, 30 \mathrm{~kg} / \mathrm{h}, 1035 \mathrm{~kg} / \mathrm{h}$, and $\infty$, respectively. According to these assumptions, the continuous MEN is the same as that for case 2. To compare storing policy only, the plant costs are not considered.

In Figure 7a, the solid lines and dotted lines denote streams coming from plant 1 and plant 2, respectively. Plant 1 is operated for $0-10 \mathrm{~h}$, and plant 2 starts operation from hour 6 to hour 16. Thus, the cycle time is $12 \mathrm{~h}$, as a result from the cyclic operation of two parallel plants. After the 10th hour, one may notice that $R_{2}$ of plant 1 and plant 2 can be connected without null time. Thus, $R_{2}$ becomes a continuous stream and no pressure vessel is needed for $R_{2}$.

Comparing the storing policies for cases 2 and 3, as shown in Figures 6 and 8, case 3 does not need a pressure vessel $R_{2}$ and the vessel/tank sizes for $R_{1}$ and $S_{1}$ are much smaller than that of case 2, as listed in Table 4 , because the available time of streams is longer than that of case 2. The storing costs of vessel $R_{1}$ and tank $S_{1}$ thus decrease dramatically, from \$529 739/ yr (case 2) to $\$ 163111 / y r$ (case 3), because of their reduced sizes.

5.4. Case 4: Two Parallel COG Plants with a Shorter Cyclic Time. In case 4, the assumption is the same as that of case 3. Therefore, the continuous MEN is still the same as that of case 2. Figure $7 \mathrm{~b}$ shows the process with two modified COG plants operated under a shorter cyclic time $(10 \mathrm{~h})$, where $R_{1}$ becomes a continuous stream and there is no need for vessel $R_{1}$. Figure 4 shows the resulting MEN configuration where the most expensive vessel $R_{1}$ is not needed. The remainders in vessel $R_{2}$ and liquid tank $S_{1}$, and the storing policies, are shown in Figure 9, and the costs data are also given in Table 4, where the TAC is reduced significantly to $\$ 131675 / \mathrm{yr}$.

5.5. Analysis of Total Annual Costs (TACs). Instead of directly minimizing the TAC for the batch MENS problem, the sequential design approach is adopted for numerical efficiency consideration. The continuous MEN is determined initially, including the mass rates of rich/lean streams flow into the mass exchangers. The streams storage policy is then resolved in Design Phase II, where the streams rates are considered as given parameters.

The effects of the lean stream rates on the costs of the MEN, as well as the streams storage, are investigated for case 2 in the following discussion. The sequential design is taken as the basic point where the lean stream rates for internal/external MSAs are $L E_{1}=749.9 \mathrm{~kg} / \mathrm{h}$ and $L E_{2}=490.54 \mathrm{~kg} / \mathrm{h}$, respectively. 


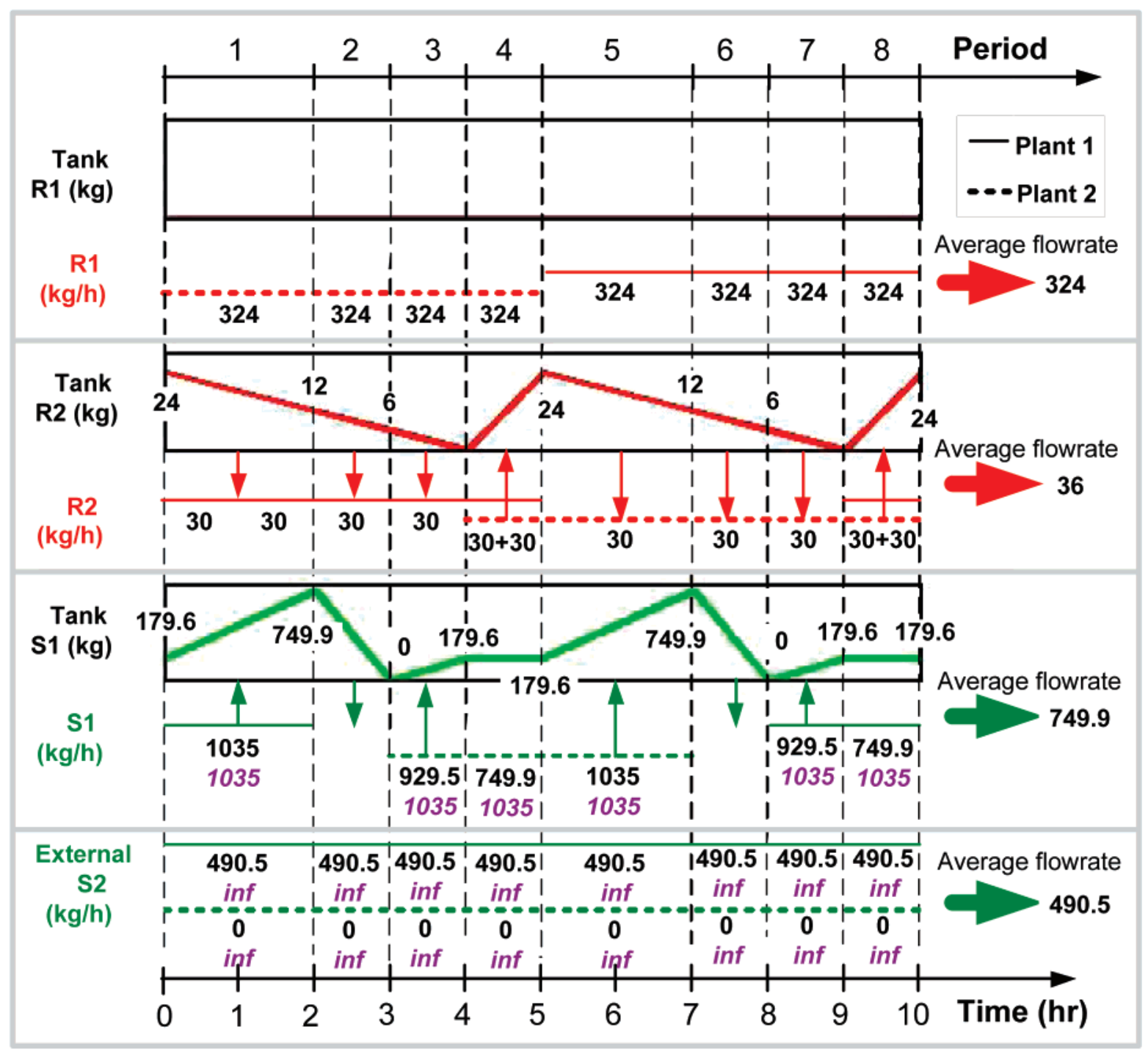

Figure 9. Remainders in vessel $R_{2}$ and liquid tank $S_{1}$ and the storing policies for case 4 .

Table 5. Effects of Lean Streams Rates on the Costs of Continuous MEN and Streams Storage for Case 2

\begin{tabular}{lllllc}
\hline & \multicolumn{2}{c}{ Zone I } & Basic Point & \multicolumn{2}{c}{ Zone II } \\
\hline$L E_{1}(\mathrm{~kg} / \mathrm{h})$ & 700 & 715 & 749.9 & 757.03 & 761.93 \\
$L E_{2}(\mathrm{~kg} / \mathrm{h})$ & 950 & 811.88 & 490.54 & 424.72 & 379.55 \\
MSA cost $(\$ / \mathrm{yr})$ & 69278 & 63010 & 48434 & 45448 & 43399 \\
$\begin{array}{l}\text { exchangers }(\$ / \mathrm{yr}) \\
\text { additional network }\end{array}$ & 40968 & 45520 & 59176 & 63728 & 68280 \\
$\quad$ cost $(\$ / \mathrm{yr})$ & & 920 & 0 & 1566 & 4069 \\
$\begin{array}{l}\text { liquid tank (\$/yr) } \\
\text { additional storage }\end{array}$ & 17350 & 17583 & 18118 & 18226 & 18300 \\
$\quad-768$ & -535 & 0 & 108 & 182 \\
$\quad$ cost $(\$ / \mathrm{yr})$ & & & & & \\
additional TAC (\$/yr) & 1868 & 385 & 0 & 1674 & 4251
\end{tabular}

The variations of costs on continuous MEN, including MSAs and exchange units, and storage for liquid lean stream $S_{1}$ are shown in Table 5 for various mass rates of the internal MSA $\left(S_{1}\right)$. Note that the mass rates of rich streams are kept at the same values in these studies, so that only the investment cost concerning liquid tank $S_{1}$ is considered. Figure 10 also shows the variations of operation cost (internal and external MSAs) and annualized investment cost (mass-exchange units), relative to the internal lean stream rate. It is reasonable that the basic point of $L E_{1}=749.9 \mathrm{~kg} / \mathrm{h}$ gives the minimal annualized total network cost, because it is the optimization result in Design Phase I.

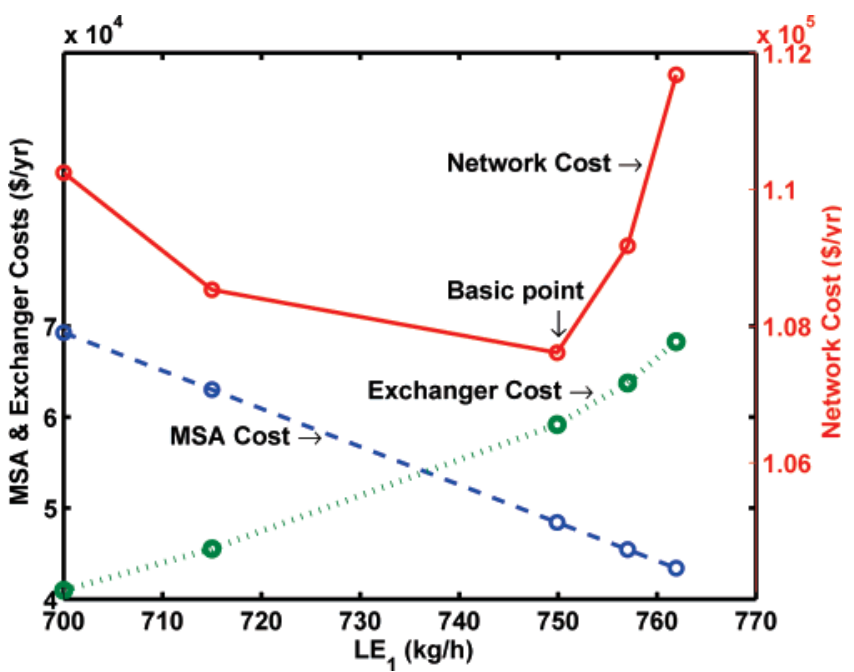

Figure 10. Network costs for various internal lean stream rates for case 2.

Table 5 also shows the additional costs of the MEN and streams storage, and the additional TAC of the overall system, respectively, in the vicinity of basic point. The variation in the additional TAC of the continuous MEN and the streams storage for different testing lean stream rates is also shown in Figure 11. Since the storage cost for liquid lean stream $S_{1}$ is weakly 


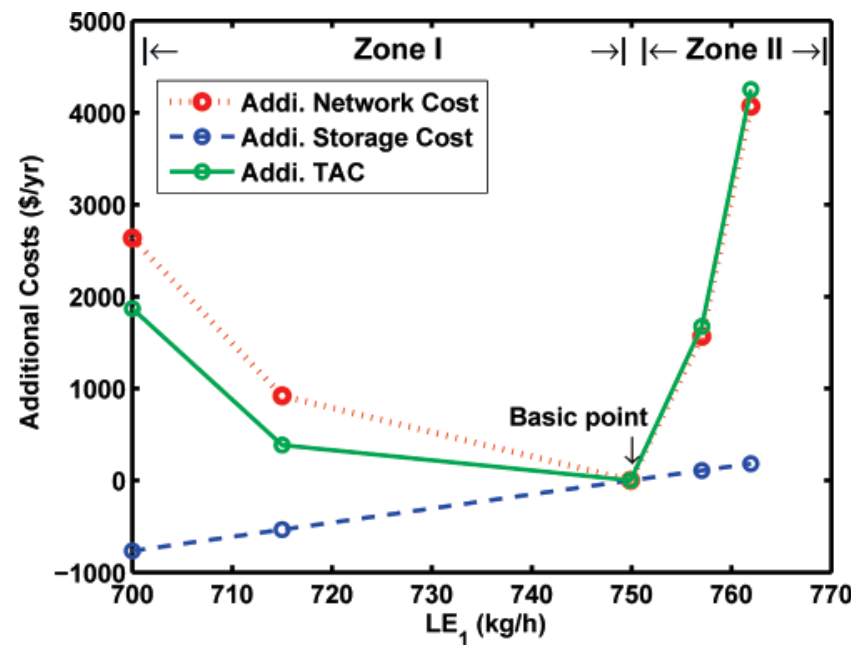

Figure 11. Additional total annual cost (TAC) for various internal lean stream rates for case 2 .

proportional to its mass rate, the additional TAC is influenced mostly by network cost. Therefore, the current optimal design determined by the sequential approach is also the solution that should be supplied by simultaneously minimizing the TAC of the overall system.

\section{Conclusion}

This paper addresses the synthesis of a mass-exchange network (MEN) for continuous treatment of time-dependent rich process streams that result from a semiconsecutive process. The operation of these streams is divided into several periods, based on the existence of process streams. A stagewise superstructure with associated pressure vessels and/or tanks for temporarily storing gas or liquid streams in surplus periods and releasing stored material during deficit intervals is presented, to represent all the alternatives of network configuration and storing policies. The synthesis problem is formulated as a mixed-integer nonlinear program (MINLP) to minimize the total annual cost (TAC) of the network and storing effort. The coke oven gases (COG) problem from the literature is supplied and is modified into different cases to demonstrate the applicability of the proposed MEN synthesis method for semiconsecutive processes.

\section{Acknowledgment}

The financial support of the Ministry of Economic Affairs (No. 95-EC-17-A-09-S1-019) and the National Science Council of ROC (No. NSC95-2214-E-002-001) is appreciated.

\section{Literature Cited}

(1) El-Halwagi, M. M.; Manousiouthakis, V. Synthesis of mass exchange networks. AIChE J. 1989, 35, 1233.
(2) El-Halwagi, M. M.; Manousiouthakis, V. Automatic synthesis of mass exchange networks with single component targets. Chem. Eng. Sci. 1990, 45, 2813.

(3) Papalexandri, K. P.; Pistikopoulos, E. N.; Floudas, C. A. Mass exchange networks for waste minimization: a simultaneous approach. Trans. Inst. Chem. Eng. 1994, 72, 279.

(4) Hallale, N.; Fraser, D. M. Capital and total cost targets for mass exchange networks. Part 1: Simple capital cost models. Comput. Chem. Eng. 2000, 23, 1661

(5) Hallale, N.; Fraser, D. M. Capital and total cost targets for mass exchange networks. Part 2: Detailed capital cost models. Comput. Chem. Eng. 2000, 23, 1681

(6) Chen, C. L.; Hung, P. S. Simultaneous synthesis of mass exchange networks for waste minimization. Comput. Chem. Eng. 2005, 29, 1561.

(7) Chen, C. L.; Hung, P. S. Retrofit of mass-exchange networks with superstructure-based MINLP formulation. Comput. Chem. Eng. 2005, 44, 7189.

(8) Chen, C. L.; Ciou, Y. J. Superstructure-Based MINLP Formulation for Synthesis of Semicontinuous Mass Exchanger Networks. Ind. Eng. Chem. Res. 2006, 45, 6728 .

(9) Wang, Y. P.; Smith, T. Wastewater minimization with flowrate constraints. Trans. Inst. Chem. Eng. 1995, A73, 889.

(10) Foo, C. Y.; Manan, Z. A.; Yunus, R. M.; Aziz, R. A. Synthesis of mass exchange network for batch processes-Part I: Utility targeting. Chem. Eng. Sci. 2004, 59, 1009.

(11) Foo, C. Y.; Manan, Z. A.; Yunus, R. M.; Aziz, R. A. Synthesis of mass exchange network for batch processes-Part II: Minimum units target and batch network design. Chem. Eng. Sci. 2005, 60, 1349.

(12) Hallale, N. Mass transfer technology for pollution prevention. In Process Design Tools for the Environment; Sikdar, S. S., El-Halwagi, M. M., Eds.; Taylor \& Francis: London, 2001; Chapter 7.

(13) Szitkai, Z.; Lelkes, Z.; Rev, E.; Fonyo, Z. Handling of removable discontinuities in MINLP models for process synthesis problems: formulations of the Kremser equation. Comput. Chem. Eng. 2002, 26, 1501.

(14) Shenoy, U. V.; Fraser, D. M. A new formulation of the Kremser equation for sizing mass exchangers. Chem. Eng. Sci. 2003, 58, 5121.

(15) Treybal, R. E. Mass Transfer Operations, 3rd Edition; McGrawHill: Singapore, 1981.

(16) Yee, T. F.; Grossmann, I. E. Simultaneous optimization models for heat integration-I. Area and energy targeting and modeling of multistream exchangers. Comput. Chem. Eng. 1990, 14, 1151.

(17) Yee, T. F.; Grossmann, I. E. Simultaneous optimization models for heat integration-II. Heat exchanger network synthesis. Comput. Chem. Eng. 1990, 14, 1165 .

(18) Chen, J. J. J. Letter to the editor: Comments on improvement on a replacement for the logarithmic mean. Chem. Eng. Sci. 1987, 42, 2488.

(19) Brooke, A.; Kendrick, D.; Meeraus, A.; Raman, R.; Rosenthal, R. E. GAMS: A User's Guide; Scientific Press: Redwood City, CA, 2003.

(20) Douglas, T. M. Conceptual Design of Chemical Processes; McGraw-Hill: New York, 1988.

Received for review December 28, 2006 Revised manuscript received July 23, 2007 Accepted July 27, 2007

IE0616803 